\title{
HOCHSCHILD COHOMOLOGY AND DEFORMATION QUANTIZATION OF AFFINE TORIC VARIETIES
}

\author{
MATEJ FILIP
}

\begin{abstract}
For an affine toric variety $\operatorname{Spec}(A)$, we give a convex geometric description of the Hodge decomposition of its Hochschild cohomology. Under certain assumptions we compute the dimensions of the Hodge summands $T_{(i)}^{1}(A)$, generalizing the existing results about the André-Quillen cohomology group $T_{(1)}^{1}(A)$. We prove that every Poisson structure on a possibly singular affine toric variety can be quantized in the sense of deformation quantization.
\end{abstract}

\section{INTRODUCTION}

The concept of deformation quantization has been appearing in the literature for many years and was established by Bayen, Flato, Frønsdal, Lichnerowicz and Sternheimer in [5]. A major result, concerning the existence of deformation quantization is Kontsevich's formality theorem [20, Theorem 4.6.2] which implies that every Poisson structure on a real manifold can be quantized, i.e., admits a star product. Kontsevich also extended the notion of deformation quantization into the algebro-geometric setting [19]. From Yekutieli's results [32, 33] it follows that on a smooth algebraic variety $X$ (under certain cohomological restrictions) every Poisson structure admits a star product. As in Kontsevich's case, the construction is canonical and induces a bijection between the set of formal Poisson structures modulo gauge equivalence and the set of star products modulo gauge equivalence (see also Van den Bergh's paper [31).

When $X=\operatorname{Spec}(A)$ is a smooth affine variety, we have the following formality theorem: there exists an $L_{\infty}$-quasi-isomorphism between the Hochschild differential graded Lie algebra $C^{\bullet}(A)[1]$ and the formal differential graded Lie algebra $H^{\bullet}(A)[1]$ (i.e., the graded Lie algebra $H^{\bullet}(A)[1]$ with trivial differential), extending the Hochschild-Kostant-Rosenberg quasi-isomorphism of the above complexes. Dolgushev, Tamarkin and Tsygan [14] proved even a stronger statement by showing that the Hochschild complex $C^{\bullet}(A)$ is formal as a homotopy Gerstenhaber algebra. Consequently, every Poisson structure on a smooth affine variety can be quantized.

Studying non-commutative deformations (also called quantizations) of toric varieties is important for constructing and enumerating noncommutative instantons (see 9], 10]), which is closely related to the computation of Donaldson-Thomas invariants on toric threefolds (see [18, [13]).

In the paper we drop the smoothness assumption and consider the deformation quantization problem for possibly singular affine toric varieties. In the singular case the Hochschild-Konstant-Rosenberg map is no longer a quasi-isomorphism and thus also the $n$-th Hochschild cohomology group is no longer isomorphic to the Hodge

2010 Mathematics Subject Classification. 13D03, 13D10, 14B05, 14B07, 14M25, 53D55.

Key words and phrases. Deformation quantization; Hochschild cohomology; Toric singularities. 
summand $H_{(n)}^{n}(A) \cong \operatorname{Hom}_{A}\left(\Omega_{A \mid k}^{n}, A\right)$. Therefore, other components of the Hodge decomposition come into play, making the problem of deformation quantization interesting from the cohomological point of view. In general many parts of the Hodge decomposition are still unknown. The case of complete intersections has been settled in [15, where Frønsdal and Kontsevich also motivated the problem of deformation quantization on singular varieties. In the toric case Altmann and Sletsjøe 44 computed the Harrison parts of the Hodge decomposition.

Deformation quantization of singular Poisson algebras does not exist in general; see Mathieu 23] for counterexamples. For known results about quantizing singular Poisson algebras we refer the reader to [29] and references therein. The associative deformation theory for complex analytic spaces was developed by Palamodov in [25] and [26]. For recent developments concerning the problem of deformation quantization in derived geometry, see 8 .

The paper is organized as follows: in Section 2 and 3 we recall definitions and some techniques for computing Hochschild cohomology. We compute the Hochschild cohomology of a reduced isolated hypersurface singularity in Proposition 3.3. Section 4 contains computations of Hochschild cohomology for toric varieties. In Theorem 4.9 we give a convex geometric description of the Hodge decomposition of the Hochschild cohomology for affine toric varieties. As an application we explicitly calculate $T_{(i)}^{1}(A)$ for all $i \in \mathbb{N}$ in the case of two and three dimensional affine toric varieties (see Propositions 4.12, 4.14). In higher dimensions we compute $T_{(i)}^{1}(A)$ for affine cones over smooth toric Fano varieties (see Theorem 4.18). In Section 5 we prove that every Poisson structure on an affine toric variety can be quantized in the sense of deformation quantization.

\section{Preliminaries}

Let $k$ be a field of characteristic 0 (in Section 5 we assume additionally that $k$ is algebraically closed) and let $A$ be an associative commutative $k$-algebra. We denote by $\mathcal{A}$ the category of local Artin $k$-algebras with the residue field $k$ (with local homomorphisms as morphisms) and by $\mathcal{S}$ we denote the category of sets.

We consider the following deformation problem: a deformation of $A$ over an Artin ring $B$ is a pair $\left(A^{\prime}, \pi\right)$, where $A^{\prime}$ is a $B$-algebra and $\pi: A^{\prime} \otimes_{B} k \rightarrow A$ is an isomorphism of $k$-algebras. Two such deformations $\left(A^{\prime}, \pi_{1}\right)$ and $\left(A^{\prime \prime}, \pi_{2}\right)$ are equivalent if there exists an isomorphism of $B$-algebras $\phi: A^{\prime} \rightarrow A^{\prime \prime}$ such that it is compatible with $\pi_{1}$ and $\pi_{2}$, i.e., such that $\pi_{1}=\pi_{2} \circ\left(\phi \otimes_{B} k\right)$. A functor that encodes this deformation problem is

$$
\begin{gathered}
\operatorname{Def}_{A}: \mathcal{A} \rightarrow \mathcal{S} \\
B \mapsto\{\text { deformations of } A \text { over } B\} / \sim .
\end{gathered}
$$

It is well-known that the differential graded Lie algebra (dgla for short) that controls this deformation problem is the Hochschild dgla $C^{\bullet}(A)[1]$, where $C^{\bullet}(A)$ is the Hochschild cochain complex, i.e., $C^{n}(A)$ is the space of $k$-linear maps $f: A^{\otimes n} \rightarrow A$ (or $A$-module homomorphisms $A \otimes A^{\otimes n} \rightarrow A$ ) with the differential given by

$$
\begin{aligned}
(d f)\left(a_{1} \otimes \cdots \otimes a_{n}\right):= & a_{1} f\left(a_{2} \otimes \cdots \otimes a_{n}\right)+ \\
& \sum_{i=1}^{n-1}(-1)^{i} f\left(a_{1} \otimes \cdots \otimes a_{i} a_{i+1} \otimes \cdots \otimes a_{n}\right)+ \\
& (-1)^{n} f\left(a_{1} \otimes \cdots \otimes a_{n-1}\right) a_{n} .
\end{aligned}
$$


The $n$-th cohomology groups of this complex is called the $n$-th Hochschild cohomology group, denoted by $\mathrm{HH}^{n}(A)$. The Lie bracket on $C^{\bullet}(A)[1]$ is coming from the Gerstenhaber bracket $[f, g]$ of $f \in C^{m}(A), g \in C^{n}(A)$, which is defined as

$$
[f, g]:=f \circ g-(-1)^{(m+1)(n+1)} g \circ f \in C^{m+n-1}(A),
$$

where

$$
\begin{gathered}
(f \circ g)\left(a_{1} \otimes \cdots \otimes a_{m+n-1}\right):= \\
\sum_{i=1}^{m}(-1)^{(i-1)(n+1)} f\left(a_{1} \otimes \cdots \otimes a_{i-1} \otimes g\left(a_{i} \otimes \cdots \otimes a_{i+n-1}\right) \otimes a_{i+n} \otimes \cdots \otimes a_{m+n-1}\right) .
\end{gathered}
$$

The Gerstenhaber bracket equips $C^{\bullet}(A)[1]$ with the structure of a dgla.

Gerstenhaber and Schack described the Hodge decomposition of the Hochschild (co-)homology that we will briefly recall (see [16] for more details). In the group ring of the permutation group $S_{n}$ one defines the shuffle $s_{i, n-i}$ to be $\sum(\operatorname{sgn} \pi) \pi$, where the sum is taken over those permutations $\pi \in S_{n}$ such that $\pi(1)<\pi(2)<\cdots<\pi(i)$ and $\pi(i+1)<\pi(i+2)<\cdots<\pi(n)$. Let $s_{n}=\sum_{i=1}^{n-1} s_{i, n-i}$. There exists orthogonal idempotents $e_{n}(i) \in S_{n}[\mathbb{Q}]$ for $i=1, \ldots, n$, whose sum is the unit element. Moreover, for $\lambda_{i}=2^{i}-2$ it holds that

$$
s_{n}=\lambda_{1} e_{n}(1)+\lambda_{2} e_{n}(2)+\cdots+\lambda_{n} e_{n}(n),
$$

which gives subcomplexes $C_{(i)}^{\bullet}(A)$, with $C_{(i)}^{n}(A)=\left\{f \in C^{n}(A) \mid f \circ s_{n}=\left(2^{i}-2\right) f\right\}$. We have

$$
\mathrm{HH}^{n}(A) \cong H_{(1)}^{n}(A) \oplus \cdots \oplus H_{(n)}^{n}(A)
$$

where $H_{(i)}^{n}(A)$ is the $n$-th cohomology of $C_{(i)}(A)$ (the part of $\mathrm{HH}^{n}(A)$ corresponding to $\left.e_{n}(i)\right)$.

It holds that $H_{n}^{(n)}(A) \cong \Omega_{A \mid k}^{n}$, the $n$-th exterior power of the module of Kähler differentials. If $A$ is smooth, we have $\operatorname{HH}^{n}(A) \cong H_{(n)}^{n}(A) \cong \operatorname{Hom}_{A}\left(\Omega_{A \mid k}^{n}, A\right)$.

Definition 1. The complex $C_{(1)}^{\bullet}(A)$ is called the Harrison complex and we will write $\operatorname{Har}^{n}(A):=H_{(1)}^{n}(A)$ for the Harrison cohomology groups.

Definition 2. A skew-symmetric Hochschild 2-cocycle $p$ that satisfies the Jacobi identity

$$
p(a \otimes p(b \otimes c))+p(b \otimes p(c \otimes a))+p(c \otimes p(a \otimes b))=0
$$

is called an (algebraic) Poisson structure (or a Poisson bracket). A commutative algebra together with a Poisson bracket that also satisfies Leibniz's law is called a Poisson algebra. Its spectrum is called an affine Poisson variety.

Using the Hodge decomposition we can equivalently define the Poisson structure as an element $p \in H_{(2)}^{2}(A)$ with $e_{3}(3)[p, p]=0$, where $e_{3}(3)$ is the orthogonal idempotent projecting $C^{3}(A)$ on $C_{(3)}^{3}(A)$ (see e.g. [25]).

Definition 3. A one-parameter formal deformation of $A$ is an associative algebra $(A[[\hbar]], *)$, such that

$$
a * b=a b(\bmod \hbar)
$$

for each $a, b \in A$. We require that $*$ is associative, $k[[\hbar]]$-bilinear and continuous, which means that

$$
\left(\sum_{m \geq 0} b_{m} \hbar^{m}\right) *\left(\sum_{n \geq 0} c_{n} \hbar^{n}\right)=\sum_{m, n \geq 0}\left(b_{m} * c_{n}\right) \hbar^{m+n} .
$$


Definition 4. We say that a Poisson structure $p \in H_{(2)}^{2}(A)$ can be quantized if there exist $\gamma_{2}, \gamma_{3}, \ldots$ in $C^{2}(A)$, such that

$$
a * b:=a b+\frac{1}{2} p(a \otimes b) \hbar+\gamma_{2}(a \otimes b) \hbar^{2}+\gamma_{3}(a \otimes b) \hbar^{3}+\cdots
$$

is a one-parameter formal deformation.

Note that when $\operatorname{Har}^{3}(A)=0$, every Poisson structure can be extended to a second order deformation (i.e. $\gamma_{2}$ always exists $\left(\bmod \hbar^{3}\right)$ since $e_{3}(3)[p, p]=e_{2}(3)[p, p]=$ $0)$.

Now we recall the standard notation in the toric setting from 4 . Let $M, N$ be mutually dual, finitely generated, free Abelian groups. We denote by $M_{\mathbb{R}}, N_{\mathbb{R}}$ the associated real vector spaces obtained via base change with $\mathbb{R}$. Given a rational, polyhedral cone $\sigma=\left\langle a_{1}, \ldots, a_{N}\right\rangle \subset N_{\mathbb{R}}$ with apex in 0 and with $a_{1}, \ldots, a_{N} \in N$ denoting its primitive fundamental generators (i.e. none of the $a_{j}$ is a proper multiple of an element of $N$ ). We define the dual cone $\sigma^{\vee}:=\left\{r \in M_{\mathbb{R}} \mid\langle\sigma, r\rangle \geq 0\right\} \subset M_{\mathbb{R}}$ and denote by $\Lambda:=\sigma^{\vee} \cap M$ the resulting semi-group of lattice points. Its spectrum $\operatorname{Spec}(k[\Lambda])$ is called an affine toric variety. For $\lambda \in \Lambda$ we denote by $x^{\lambda}$ the monomial corresponding to $\lambda$. Since $\Lambda$ is saturated, $\operatorname{Spec}(k[\Lambda])$ is normal (see e.g. [11, Theorem 1.3.5]).

Definition 5. A variety $X$ is called $\mathbb{Q}$-Gorenstein if the double dual of some tensor product of $\omega_{X}$ is an invertible sheaf on $X$.

The following facts about toric $\mathbb{Q}$-Gorenstein varieties can be found in 1 , Section 6.1]. For an affine toric variety given by the cone $\sigma=\left\langle a_{1}, \ldots, a_{N}\right\rangle$ we have that $X$ is $\mathbb{Q}$-Gorenstein if and only if there exists a primitive element $R^{*} \in M$ and a natural number $g \in \mathbb{N}$ such that $\left\langle a_{j}, R^{*}\right\rangle=g$ for each $j=1, \ldots, N$. $X$ is Gorenstein if and only if additionally $g=1$. In particular, toric $\mathbb{Q}$-Gorenstein singularities are obtained by putting a lattice polytope $P \subset \mathbb{A}$ into the affine hyperplane $\mathbb{A} \times\{g\} \subset$ $N_{\mathbb{R}}:=\mathbb{A} \times \mathbb{R}$ and defining $\sigma:=\operatorname{Cone}(P)$, the cone over $P$. Then the canonical degree $R^{*}$ equals $(\underline{0}, 1)$.

\section{André-Quillen COHOMOlOGy}

In this section we recall the geometric approach (using the cotangent complex) for computing the Hochschild cohomology. As an application we compute the Hochschild cohomology of a reduced isolated hypersurface singularity, which will give a more complete view on the results that we will obtain in the next section (see Example 3).

We will briefly recall the construction of the cotangent complex (for more details see [22]).

Definition 6. A dg-algebra $R$ with differential $s$ is called semifree if:

- The underlying graded algebra is a polynomial algebra $k\left[x_{i} \mid i \in I\right]$, where the degree of $x_{i}$ may vary.

- There exists a filtration

$$
\emptyset=I(0) \subset I(1) \subset \cdots, \cup_{n \in \mathbb{N}} I(n)=I,
$$

such that $s\left(x_{i}\right) \in k\left[x_{j} \mid j \in I(n)\right]$ for every $i \in I(n+1)$. 
A $k$-semifree resolution of an algebra $A$ is a surjective quasi-isomorphism $R \rightarrow A$, where $R$ is a semifree $k$-dg-algebra. Note that a $k$-semifree resolution always exists. The corresponding complex of the $A$-dg module $\Omega_{R \mid k} \otimes_{R} A$ gives us the element $\mathbb{L}_{A \mid k}$ in the derived category $\mathrm{D}\left(\mathfrak{M o d}_{A}\right)$. We call $\mathbb{L}_{A \mid k}$ the cotangent complex. It is independent of the choice of the $k$-semifree resolution.

We have a quasi-isomorphism between $\left(\mathbb{L}_{A \mid k}\right)[1]$ and $C_{\bullet}^{(1)}(A)$ (see e.g. [21, Proposition 4.5.13]). Moreover, the derived exterior powers $\wedge^{i} \mathbb{L}_{A \mid k}$ (see [21, Section 3.5.4] for definitions) give us the following proposition.

Proposition 3.1. There exists a quasi-isomorphism between $\wedge^{i}\left(\mathbb{L}_{A \mid k}\right)[i]$ and $C_{\bullet}^{(i)}(A)$.

Proof. See [21, Proposition 4.5.13].

Example 1. Let $X=\operatorname{Spec}(A)$ be a reduced hypersurface, where

$$
A=k\left[x_{1}, \ldots, x_{N}\right] /\left(f\left(x_{1}, \ldots, x_{N}\right)\right) .
$$

Let us denote $S=k\left[x_{1}, \ldots, x_{N}\right]$. The $i$-th derived exterior power $\wedge^{i} \mathbb{L}_{A \mid k}$ is isomorphic to the complex

$$
0 \rightarrow A \stackrel{\wedge d f}{\longrightarrow} \Omega_{S \mid k}^{1} \otimes_{S} A \stackrel{\wedge d f}{\longrightarrow} \cdots \stackrel{\wedge d f}{\longrightarrow} \Omega_{S \mid k}^{i} \otimes_{S} A \rightarrow 0,
$$

where $\Omega_{S \mid k}^{i} \otimes_{S} A$ is the degree 0 term. We can prove (1) by first computing the cotangent complex and since it has only two non-zero terms, we can use 28, Chapter 4] (see also [17]) to compute the derived exterior powers.

Definition 7. The $n$-th homology group of $\wedge^{i} \mathbb{L}_{A \mid k}$ is called the $n$-th (higher) AndréQuillen homology group and denoted by $T_{n}^{(i)}(A)$. The $n$-th cohomology group of $\operatorname{Hom}_{A}\left(\wedge^{i} \mathbb{L}_{A \mid k}, A\right)$ is called the $n$-th (higher) André-Quillen cohomology group and denoted by $T_{(i)}^{n}(A)$.

In particular, from Proposition 3.1 we have an isomorphism of groups

$$
T_{(1)}^{n-1}(A) \cong H_{(1)}^{n}(A)=\operatorname{Har}^{n}(A),
$$

or more generally $T_{(i)}^{n-i}(A) \cong H_{(i)}^{n}(A)$, for each $i=1, \ldots, n$. For a smooth algebra $A$ we have

$$
\mathrm{HH}^{n}(A) \cong H_{(n)}^{n}(A) \cong T_{(n)}^{0}(A)
$$

and thus we see that for $j>0$ the modules $T_{(i)}^{j}(A)$ (and similarly for $T_{j}^{(i)}(A)$ ) have support on the singular locus.

The next result relates André-Quillen cohomology groups with Ext groups.

Lemma 3.2. Let $X=\operatorname{Spec}(A)$ be smooth in codimension $d$. For each $i \geq 1$ and $0 \leq j \leq d+1$, we have $T_{(i)}^{j}(A) \cong \operatorname{Ext}_{A}^{j}\left(\Omega_{A \mid k}^{i}, A\right)$.

Proof. Since each term of $\wedge^{i} \mathbb{L}_{A \mid k}$ is a projective $A$-module for each $i \geq 1$, we have a Künneth spectral sequence:

$$
E_{2}^{p, q}=\operatorname{Ext}_{A}^{p}\left(T_{q}^{(i)}(A), A\right) \Rightarrow T_{(i)}^{p+q}(A) .
$$

The modules $T_{q}^{(i)}(A)$ have support on the singular locus for $q \geq 1$. Since $A$ is smooth in codimension $d$, we have $\operatorname{Ext}_{A}^{p}\left(T_{q}^{(i)}(A), A\right)=0$ for $q \geq 1$ and $p=$ $0,1, \ldots, d$. 
Proposition 3.3. Let $A$ be a reduced isolated hypersurface singularity in $\mathbb{A}^{N}$. We have

$$
\mathrm{HH}^{n}(A) \cong \begin{cases}\operatorname{Hom}_{A}\left(\Omega_{A \mid k}^{n}, A\right) \oplus A /\left(\frac{\partial f}{\partial x_{1}}, \frac{\partial f}{\partial x_{2}}, \ldots, \frac{\partial f}{\partial x_{N}}\right) & \text { if } n<N \\ A /\left(\frac{\partial f}{\partial x_{1}}, \frac{\partial f}{\partial x_{2}}, \ldots, \frac{\partial f}{\partial x_{N}}\right) & \text { if } n \geq N\end{cases}
$$

Proof. We use Example 1. The perfect pairing $\Omega_{S \mid k}^{j} \otimes_{S} \Omega_{S \mid k}^{N-j} \rightarrow \Omega_{S \mid k}^{N} \cong S$ induces an isomorphism of complexes $\operatorname{Hom}_{A}\left(\wedge^{N} \mathbb{L}_{A \mid k}, A\right)[-N] \cong \wedge^{N} \mathbb{L}_{A \mid k}$. By Michler's result in [24] the only nonzero homology groups of $\wedge^{N} \mathbb{L}_{A \mid k}$ are the zeroth and first, both isomorphic to $A /\left(\frac{\partial f}{\partial x_{1}}, \frac{\partial f}{\partial x_{2}}, \ldots, \frac{\partial f}{\partial x_{N}}\right)$. Note that $\Omega_{S \mid k}^{l}=0$ holds for $l \geq N+1$ and thus for $i \geq N$ we have that

$$
T_{(i)}^{j}(A) \cong \begin{cases}A /\left(\frac{\partial f}{\partial x_{1}}, \frac{\partial f}{\partial x_{2}}, \ldots, \frac{\partial f}{\partial x_{N}}\right) & \text { if } j=i-1, i \\ 0 & \text { otherwise. }\end{cases}
$$

By [24] we also know that $\wedge^{k} \mathbb{L}_{A \mid k}$ is quasi-isomorphic to $\Omega_{A \mid k}^{k}$ for $k \leq N-1$. Thus we can easily see that $\operatorname{Ext}_{A}^{j}\left(\Omega_{A \mid k}^{k}, A\right)=0$, if $k \leq N-1$ and $j \neq 0, k-1, k$. Moreover, in the decomposition $\operatorname{Ext}_{A}^{1}\left(\Omega_{A \mid k}^{n-1}, A\right) \oplus \cdots \oplus \operatorname{Ext}_{A}^{n-1}\left(\Omega_{A \mid k}^{1}, A\right)$ only one direct summand is nonzero and isomorphic to $\Omega_{A \mid k}^{N} \cong A /\left(\frac{\partial f}{\partial x_{1}}, \frac{\partial f}{\partial x_{2}}, \ldots, \frac{\partial f}{\partial x_{N}}\right)$. Lemma 3.2 and the Hodge decomposition conclude the proof.

\section{Hochschild COHOMOLOGY OF TORIC VARIETIES}

From now on we will restrict ourself in the case of toric varieties and try to simplify the results using the lattice grading that comes with toric varieties. The convex geometric description of Harrison cohomology groups of an affine toric variety was given in [4]. We generalize this result to the case of Hochschild cohomology groups.

Let $A=\oplus_{i \in \mathbb{Z}} A_{i}$ be a graded $k$-algebra. If $a_{0}, \ldots, a_{p}$ are homogenous elements, define the weight of $a_{0} \otimes \cdots \otimes a_{p} \in A^{\otimes p+1}$ to be $w=\sum\left|a_{i}\right|$, where $\left|a_{i}\right|=j$ means that $a_{i} \in A_{j}$. This makes the tensor product $A^{\otimes p+1}$ into a graded $k$-module. Since differentials preserve the weight, this equip both $\operatorname{HH}_{p}(A)$ and $\operatorname{HH}^{p}(A)$ with the structure of graded $k$-modules.

4.1. The Hochschild complex in the toric case. Definitions and statements in this subsection already appeared in [4] for $i=1$. We give a generalization for arbitrary $i \geq 1$.

In the case when $\operatorname{Spec}(A)$ is an affine toric variety there exists $M$-grading on $A$. Let $A=k[\Lambda]=k\left[\sigma^{\vee} \cap M\right]$.

Definition 8. We say that an element $f \in C^{n}(A)$ has degree $R \in M$ if $f$ maps an element with weight $w$ to an element of degree $R+w$ in $A$. This means that $f$ is of the form $f\left(x^{\lambda_{1}} \otimes \cdots \otimes x^{\lambda_{n}}\right)=f_{0}\left(\lambda_{1}, . ., \lambda_{n}\right) x^{R+\lambda_{1}+\cdots+\lambda_{n}}$. We need to take care that the expression is well defined, i.e., that $f_{0}\left(\lambda_{1}, \ldots, \lambda_{n}\right)=0$ for $R+\lambda_{1}+\cdots \lambda_{n} \notin \Lambda$ (in the following we will also use $R+\lambda_{1}+\cdots \lambda_{n} \ngtr 0$ since we can look on $M$ as a partially ordered set where positive elements lie in the cone $\Lambda)$. Let $C^{n, R}(A)$ denote the degree $R$ elements of $C^{n}(A)$ and let $C_{(i)}^{n, R}(A)$ denote the degree $R$ elements of $C_{(i)}^{n}(A)$.

We would like to understand the space $C^{n, R}(A)$ better and the following definition will be useful. 
Definition 9. $L \subset \Lambda$ is said to be monoid-like if for all elements $\lambda_{1}, \lambda_{2} \in L$ the relation $\lambda_{1}-\lambda_{2} \in \Lambda$ implies $\lambda_{1}-\lambda_{2} \in L$. Moreover, a subset $L_{0} \subset L$ of a monoid-like set is called full if $\left(L_{0}+\Lambda\right) \cap L=L_{0}$.

For any subset $P \subset \Lambda$ and $n \geq 1$ we introduce $S_{n}(P):=\left\{\left(\lambda_{1}, \ldots, \lambda_{n}\right) \in\right.$ $\left.P^{n} \mid \sum_{v=1}^{n} \lambda_{v} \in P\right\}$. If $L_{0} \subset L$ are as in the previous definition, then this gives rise to the following vector spaces $(1 \leq i \leq n)$ :

$C_{(i)}^{n}\left(L, L \backslash L_{0} ; k\right):=\left\{\varphi: S_{n}(L) \rightarrow k \mid \varphi \circ s_{n}=\left(2^{i}-2\right) \varphi, \varphi\right.$ vanishes on $\left.S_{n}\left(L \backslash L_{0}\right)\right\}$, which turn into a complex with the differential

$$
\begin{gathered}
d^{n}: C_{(i)}^{n-1}\left(L, L \backslash L_{0} ; k\right) \rightarrow C_{(i)}^{n}\left(L, L \backslash L_{0} ; k\right), \\
\left(d^{n} \varphi\right)\left(\lambda_{1}, \ldots, \lambda_{n}\right):= \\
\varphi\left(\lambda_{2}, \ldots, \lambda_{n}\right)+\sum_{i=1}^{n-1}(-1)^{i} \varphi\left(\lambda_{1}, \ldots, \lambda_{i}+\lambda_{i+1}, \ldots, \lambda_{n}\right)+(-1)^{n} \varphi\left(\lambda_{1}, \ldots, \lambda_{n-1}\right) .
\end{gathered}
$$

We will see that this complexes will give us a description of a degree $-R \in M$ part of $H_{(i)}^{n}(A)$.

Definition 10. By $H_{(i)}^{n}\left(L, L \backslash L_{0} ; k\right)$ we denote the cohomology groups of the above complex $C_{(i)}^{\bullet}\left(L, L \backslash L_{0} ; k\right)$.

\section{Lemma 4.1.}

$$
C_{(i)}^{n,-R}(A) \cong C_{(i)}^{n}(\Lambda, \Lambda \backslash(R+\Lambda) ; k) .
$$

Proof. For $f \in C_{(i)}^{n,-R}(A)$, we have $f\left(x^{\lambda_{1}} \otimes \cdots \otimes x^{\lambda_{n}}\right)=f_{0}\left(\lambda_{1}, . ., \lambda_{n}\right) x^{\lambda_{1}+\cdots+\lambda_{n}-R}$ and then the isomorphism is given by $f \mapsto f_{0}$.

It is a trivial check that Hochschild differentials respect the grading given by the degrees $R \in M$. Thus we get the Hochschild subcomplex $C_{(i)}^{\bullet,-R}$ and we denote the corresponding cohomology groups by $H_{(i)}^{n,-R}(A) \cong T_{(i)}^{n-i,-R}(A)$.

From definitions it follows that $C_{(i)}^{n}(A)=\oplus_{R} C_{(i)}^{n,-R}(A), C^{n}(A)=\oplus_{R} C^{n,-R}(A)$ and $H_{(i)}^{n}(A)=\oplus_{R} H_{(i)}^{n,-R}(A), \mathrm{HH}^{n}(A)=\oplus_{R} \mathrm{HH}^{n,-R}(A)$.

Proposition 4.2. Let $R \in M$ and let $A=k[\Lambda]$. We have

$$
T_{(i)}^{n-i,-R}(A) \cong H_{(i)}^{n}(\Lambda, \Lambda \backslash(R+\Lambda) ; k) .
$$

Proof. We use Lemma 4.1 and the decomposition of the Hochschild cohomology.

Remark 1. We will also use the positive grading

$$
T_{(i)}^{n-i, R}(A) \cong H_{(i)}^{n}(\Lambda, \Lambda \backslash(-R+\Lambda) ; k) .
$$

Poisson structures lie in $T_{(2)}^{0}(A)$, which is non-zero for positive degrees. 
4.2. A double complex of convex sets. In this subsection we follow the paper [4] verbatim. Arguments mention in [4] in the case $i=1$ work also for arbitrary $i \geq 1$ using the definitions from Subsection 4.1

Let $\sigma=\left\langle a_{1}, \ldots, a_{N}\right\rangle$. For $\tau \subset \sigma$ let us define the convex sets introduced in [4]:

$$
K_{\tau}^{R}:=\Lambda \cap\left(R-\operatorname{int} \tau^{\vee}\right) .
$$

The above convex sets admit the following properties:

- $K_{0}^{R}=\Lambda$ and $K_{a_{j}}^{R}=\left\{r \in \Lambda \mid\left\langle a_{j}, r\right\rangle<\left\langle a_{j}, R\right\rangle\right\}$ for $j=1, \ldots, N$.

- For $\tau \neq 0$ the equality $K_{\tau}^{R}=\cap_{a_{j} \in \tau} K_{a_{j}}^{R}$ holds.

- $\Lambda \backslash(R+\Lambda)=\cup_{j=1}^{N} K_{a_{j}}^{R}$.

We have the following double complexes $C_{(i)}\left(K_{\bullet}^{R} ; k\right)$ for each $i \geq 1$. We define $C_{(i)}^{q}\left(K_{\tau}^{R} ; k\right):=C_{(i)}^{q}\left(K_{\tau}^{R}, \emptyset ; k\right)$ and

$$
C_{(i)}^{q}\left(K_{p}^{R} ; k\right):=\oplus_{\tau \leq \sigma, \operatorname{dim} \tau=p} C_{(i)}^{q}\left(K_{\tau}^{R} ; k\right) \quad(0 \leq p \leq \operatorname{dim} \sigma) .
$$

The differentials $\delta^{p}: C_{(i)}^{q}\left(K_{p}^{R}\right) \rightarrow C_{(i)}^{q}\left(K_{p+1}^{R} ; k\right)$ are defined in the following way: we are summing (up to a sign) the images of the restriction map $C_{(i)}^{q}\left(K_{\tau}^{R} ; k\right) \rightarrow$ $C_{(i)}^{q}\left(K_{\tau^{\prime}}^{R} ; k\right)$, for any pair $\tau \leq \tau^{\prime}$ of $p$ and $(p+1)$-dimensional faces, respectively. The sign arises from the comparison of the (pre-fixed) orientations of $\tau$ and $\tau^{\prime}$ (see also [11, pg. 580] for more details).

Example 2. The map $\delta: \oplus_{j=1}^{N} C_{(i)}^{q}\left(K_{a_{j}}^{R} ; k\right) \rightarrow \oplus_{\left\langle a_{j}, a_{k}\right\rangle \leq \sigma} C_{(i)}^{q}\left(K_{a_{j}}^{R} \cap K_{a_{k}}^{R} ; k\right)$ is simply given by: $\left(f_{1}, \ldots ., f_{N}\right)$ gets mapped to $f_{j}-f_{k} \in C_{(i)}^{q}\left(K_{a_{j}}^{R} \cap K_{a_{k}}^{R} ; k\right)$.

The following results (obtained in [4] for $i=1$ ) can also be generalized to $i>1$ :

Lemma 4.3. The canonical k-linear map $C_{(i)}^{q}(\Lambda, \Lambda \backslash(R+\Lambda) ; k) \rightarrow C_{(i)}^{q}\left(K_{\bullet}^{R} ; k\right)$ is a quasi-isomorphism, i.e., a resolution of the first vector space.

Proof. For $r \in \Lambda \subset M$ we define the $k$-vector space

$$
V_{(i)}^{q}(r):=\left\{\varphi:\left\{\left(\lambda_{1}, \ldots, \lambda_{q}\right) \in \Lambda^{q} \mid \sum_{v=1}^{q} \lambda_{v}=r\right\} \rightarrow k \mid \varphi \circ s_{n}=\left(2^{i}-2\right) \varphi\right\} .
$$

and the rest follows as in 4 .

Proposition 4.4. $T_{(i)}^{n-i,-R}(A)=H^{n}\left(\operatorname{tot} \bullet\left(C_{(i)}\left(K_{\bullet}^{R} ; k\right)\right)\right)$ for $1 \leq i \leq n$.

Proof. We prove the Proposition using first the differentials $d^{n}$ and Lemma 4.3 and then the differentials $\delta^{p}$.

Corollary 4.5. Let $i \geq 1$ be a fixed integer. For $q \geq i$ and $p \geq 0$ there is a spectral sequence

$$
E_{1}^{p, q}=\oplus_{\tau \leq \sigma, \operatorname{dim} \tau=p} H_{(i)}^{q}\left(K_{\tau}^{R} ; k\right) \Rightarrow T_{(i)}^{p+q-i,-R}(A)=H_{(i)}^{p+q,-R}(A) .
$$

Proof. We use first the differentials $\delta^{p}$ and then the differentials $d^{n}$.

Proposition 4.6. If $\tau \leq \sigma$ is a smooth face, then $H_{(i)}^{q}\left(K_{\tau}^{R} ; k\right)=0$ for $q \geq i+1$. 
Proof. Let $r(\tau)$ be an arbitrary element of $\operatorname{int}\left(\sigma^{\vee} \cap \tau^{\perp}\right) \cap M$, i.e., $\tau=\sigma \cap r(\tau)^{\perp}$. We define $R_{g}:=R-g \cdot r(\tau)$, where $g \in \mathbb{Z}$ and we show (with the same idea as in [4]) that

$$
T_{(i)}^{q+\operatorname{dim} \tau-i}\left(-R_{g}\right)=H_{(i)}^{q}\left(K_{\tau}^{R} ; k\right) \text { for } g \gg 0,
$$

for $q \geq i+1$

Let $T_{(i)}^{n}(\tau):=T_{(i)}^{n}\left(\operatorname{Spec}\left(k\left[\tau^{\vee} \cap M\right]\right)\right)$ and similarly $T_{(i)}^{n}(\sigma):=T_{(i)}^{n}(A)$. We have

$$
T_{(i)}^{n}(\sigma) \otimes_{k\left[\sigma^{\vee} \cap M\right]} k\left[\sigma^{\vee} \cap M\right]_{x^{r(\tau)}}=T_{(i)}^{n}(\tau)=0 \text { for } n \geq 1,
$$

since $k\left[\tau^{\vee} \cap M\right]$ equals the localization of $k\left[\sigma^{\vee} \cap M\right]$ by the element $x^{r(\tau)}$. The last equality holds because $\tau$ is a smooth face. From (5) we see that any element of $T_{(i)}^{q+\operatorname{dim} \tau-i}\left(-R_{g}\right) \subset T_{(i)}^{q+\operatorname{dim} \tau-i}$ will be killed by some power of $x^{r(\tau)}$, which implies that $H_{(i)}^{q}\left(K_{\tau}^{R} ; k\right)=0$ by (4).

4.3. The Hochschild cohomology in degree $R \in M$. The results in this subsection do not follow immediately from [4] as in Subsection 4.2. Quasi-linear functions (see [4, Definition 4.1]) defined on the convex sets $K_{\tau}^{R}$ play an important role in describing $T_{(1)}^{1}(-R)$. In this subsection we show that multi-additive functions (see Definition 11) are the right generalization for describing $T_{(i)}^{1}(-R)$ for $i \geq 1$. The main result in this subsection is Theorem 4.9, which is a generalization of [4, Proposition 5.2].

We would like to better understand $H_{(n)}^{n}\left(K_{\tau}^{R} ; k\right)$ for $\tau \leq \sigma$. These computations are easier then computations for $H_{(i)}^{n}\left(K_{\tau}^{R} ; k\right), i \neq n$, because in the case $i=n$ we do not have coboundaries.

Definition 11. We say that $f \in C_{(n)}^{n}\left(L, L \backslash L_{0} ; k\right)$ is multi-additive if it is additive on every component, provided that the sum of all entries lies in $L$. Being additive in the first component means $f\left(a+b, \lambda_{2}, \ldots, \lambda_{n}\right)=f\left(a, \lambda_{2}, \ldots, \lambda_{n}\right)+f\left(b, \lambda_{2}, \ldots, \lambda_{n}\right)$, with $a+b+\lambda_{1}+\cdots+\lambda_{n} \in L$. We denote

$$
\bar{C}_{(n)}^{n}\left(L, L \backslash L_{0} ; k\right):=\left\{f \in C_{(n)}^{n}\left(L, L \backslash L_{0} ; k\right) \mid f \text { is multi-additive }\right\} .
$$

In the case $n=1$ it holds trivially that $H_{(1)}^{1}\left(L, L \backslash L_{0} ; k\right)=\bar{C}_{(1)}^{1}\left(L, L \backslash L_{0} ; k\right)$. Some additional effort is necessary to show this for $n>1$.

Proposition 4.7. We have

$$
H_{(n)}^{n}\left(L, L \backslash L_{0} ; k\right)=\bar{C}_{(n)}^{n}\left(L, L \backslash L_{0} ; k\right)
$$

for all $n \geq 1$.

Proof. That every multi-additive function $f \in C_{(n)}^{n}\left(L, L \backslash L_{0} ; k\right)$ satisfies $d f=0$ is obvious by definition of $d$. For the other direction we use the following computation:

$$
\begin{gathered}
\sum_{\sigma} d f\left(\lambda_{\sigma^{-1}(1)}, \ldots, \lambda_{\sigma^{-1}(n+1)}\right)= \\
n !\left(f\left(\lambda_{1}, \lambda_{3}, \lambda_{4}, \ldots, \lambda_{n+1}\right)+f\left(\lambda_{2}, \lambda_{3}, \lambda_{4}, \ldots, \lambda_{n+1}\right)-f\left(\lambda_{1}+\lambda_{2}, \lambda_{3}, \lambda_{4}, \ldots, \lambda_{n+1}\right)\right),
\end{gathered}
$$

where the sum is taken over all permutations $\sigma \in S_{n+1}$ such that $\sigma(1)<\sigma(2)$ (similarly as in the proof of Loday [21, Proposition 1.3.12]).

The next Proposition will give us very useful formulas for $H_{(n)}^{n}\left(K_{\tau}^{R} ; k\right)$. 
Proposition 4.8. Let $\tau \leq \sigma$ be a smooth face. The injections $\bar{C}_{(n)}^{n}\left(\operatorname{Span}_{k} K_{\tau}^{R} ; k\right) \rightarrow$ $\bar{C}_{(n)}^{n}\left(K_{\tau}^{R} ; k\right)$ are isomorphisms. Moreover, $\operatorname{Span}_{k} K_{\tau}^{R}=\cap_{a_{j} \in \tau} \operatorname{Span}_{k} K_{a_{j}}^{R}$, and we have

$$
\operatorname{Span}_{k} K_{a_{j}}^{R}= \begin{cases}0 & \text { if }\left\langle a_{j}, R\right\rangle \leq 0 \\ \left(a_{j}\right)^{\perp} & \text { if }\left\langle a_{j}, R\right\rangle=1 \\ M \otimes_{\mathbb{Z}} k & \text { if }\left\langle a_{j}, R\right\rangle \geq 2\end{cases}
$$

Proof. The case $n=1$ was proved in [4, Proposition 4.2]. We will generalize it to the case $n=2$. The generalization to other $n$ is then immediate.

Let $f \in \bar{C}_{(2)}^{2}\left(K_{\tau}^{R} ; k\right)$. We want to show that $f \in \bar{C}_{(2)}^{2}\left(\operatorname{Span}_{k} K_{\tau}^{R} ; k\right)$. Without loss of generality we can assume that $\tau=\left\langle a_{1}, \ldots, a_{m}\right\rangle$, with $\left\langle a_{i}, R\right\rangle \geq 2$ for $i=1, \ldots, l$ and $\left\langle a_{j}, R\right\rangle=1$ for $j=l+1, \ldots, m$, since if $R$ was non-positive on any of the generators of $\tau$, then $K_{\tau}^{R}$ would be empty.

By the smoothness of $\tau$ there exist elements $r_{1}, \ldots, r_{l}$ such that $\left\langle r_{i}, a_{k}\right\rangle=\delta_{i k}$ for $1 \leq i \leq l$ and $1 \leq k \leq m$. Hence it holds that

$$
f\left(s_{v}, s_{w}\right)=\sum_{i=1}^{l} \sum_{u=1}^{l}\left\langle a_{i}, s_{v}\right\rangle\left\langle a_{u}, s_{w}\right\rangle f\left(r_{i}, r_{u}\right)+f\left(p_{v}, p_{w}\right),
$$

for $s_{v}, s_{w} \in K_{\tau}^{R}, p_{v}:=s_{v}-\sum_{i=1}^{l}\left\langle a_{i}, s_{v}\right\rangle r_{i} \in \tau^{\perp} \cap M$ and $p_{w}:=s_{w}-\sum_{i=1}^{l}\left\langle a_{i}, s_{w}\right\rangle r_{i} \in$ $\tau^{\perp} \cap M$. We can easily show that $\sum_{v} \sum_{w} f\left(s_{v}, s_{w}\right)$ does depend only on $s_{1}:=\sum_{v} s_{v}$ and $s_{2}:=\sum_{w} s_{w}$, and not on the summands themselves. Then, $f\left(s_{1}, s_{2}\right)$ may be defined as this value. The second claim follows as in [4] by $\cap_{a_{i} \in \tau} \operatorname{Span}_{k} K_{a_{i}}^{R}=$ $\cap_{j=l+1}^{k}\left(a_{j}\right)^{\perp}=\operatorname{Span}_{k}\left(\tau^{\perp}, r_{1}, \ldots, r_{l}\right)=\operatorname{Span}_{k} K_{\tau}^{R}$.

We write shortly $M_{k}$ (resp. $N_{k}$ ) for $M \otimes_{\mathbb{Z}} k$ (resp. $N \otimes_{\mathbb{Z}} k$ ).

Remark 2. Note that 0 and 1-dimensional faces are always smooth. For $\tau=0$ we obtain that $\bar{C}_{(i)}^{i}(\Lambda ; k) \cong \bar{C}_{(i)}^{i}\left(\operatorname{Span}_{k} \Lambda ; k\right) \cong \bar{C}_{(i)}^{i}\left(M_{k} ; k\right)$. Thus if $\sigma=\left\langle a_{1}, \ldots, a_{N}\right\rangle \subset$ $M_{k} \cong k^{n}$, then $f \in \bar{C}_{(i)}^{i}(\Lambda ; k)$ is completely determined by the values $f\left(s_{k_{1}}, \ldots, s_{k_{i}}\right)$, for $1 \leq k_{1}<\cdots<k_{i} \leq n$, where $s_{1}, \ldots, s_{n} \in \Lambda$ are linearly independent ( $k$-basis in $\left.k^{n}\right)$.

Let $E$ be the minimal set that generates the semigroup $\Lambda:=\sigma^{\vee} \cap M$. We write $E_{j}^{R}:=E \cap K_{a_{j}}^{R}, E_{j k}^{R}:=E \cap K_{a_{j}}^{R} \cap K_{a_{k}}^{R}$ for a 2-face $\left\langle a_{j}, a_{k}\right\rangle \leq \sigma$ and $E_{\tau}^{R}:=\cap_{a_{j} \in \tau} E_{j}^{R}$ for faces $\tau \leq \sigma$.

Theorem 4.9. Let $X=\operatorname{Spec}(A)$ be an affine toric variety that is smooth in codimension $d$. Let $i \geq 1$ be a fixed integer. Then $k$-th cohomology group of the complex $0 \rightarrow \bar{C}_{(i)}^{i}\left(M_{k} ; k\right) \rightarrow \oplus_{j} \bar{C}_{(i)}^{i}\left(\operatorname{Span}_{k} E_{j}^{R} ; k\right) \rightarrow \cdots \rightarrow \oplus_{\tau \leq \sigma, \operatorname{dim} \tau=d+1} \bar{C}_{(i)}^{i}\left(\operatorname{Span}_{k} E_{\tau}^{R} ; k\right)$ equals $T_{(i)}^{k,-R}(A)$, for $k=0, \ldots, d\left(\bar{C}_{(i)}^{i}\left(M_{k} ; k\right)\right.$ is the degree 0 term $)$.

Moreover, if $X$ is an isolated singularity (i.e. $\operatorname{dim}(X)=d+1$ ), then

$T_{(i)}^{k,-R}(A)= \begin{cases}\operatorname{Coker}\left(\oplus_{\tau \leq \sigma, \operatorname{dim} \tau=d} \bar{C}_{(i)}^{i}\left(K_{\tau}^{R} ; k\right) \rightarrow \bar{C}_{(i)}^{i}\left(K_{\sigma}^{R} ; k\right)\right) & \text { if } k=\operatorname{dim}(X) \\ H_{(i)}^{k-\operatorname{dim}(X)+i}\left(K_{\sigma}^{R} ; k\right) & \text { if } k \geq \operatorname{dim}(X)+1\end{cases}$

Proof. By Corollary 4.5 we have

$$
E_{1}^{p, q}=\oplus_{\tau \leq \sigma, \operatorname{dim} \tau=p} H_{(i)}^{q}\left(K_{\tau}^{R} ; k\right) \Rightarrow T_{(i)}^{p+q-i,-R}(A)=H_{(i)}^{p+q,-R}(A),
$$

for $q \geq i$ and $p \geq 0$. By the assumption $j$-dimensional faces are smooth for $j \leq d$. From Proposition 4.6] it follows that $E_{1}^{0, q}=E_{1}^{1, q}=\cdots=E_{1}^{d, q}=0$, for $q \geq i+1$. 
Thus $E_{2}^{p, i}=E_{\infty}^{p, i}=\oplus_{\tau \leq \sigma, \operatorname{dim} \tau=p} H_{(i)}^{i}\left(K_{\tau}^{R} ; k\right)$ for $d+1 \geq p \geq 1$. It follows that $T_{(i)}^{k,-R}(A)$ is the $k$-th cohomology group of the complex

$$
H_{(i)}^{i}(\Lambda ; k) \rightarrow \oplus_{j} H_{(i)}^{i}\left(K_{a_{j}}^{R} ; k\right) \rightarrow \cdots \rightarrow \oplus_{\tau \leq \sigma, \operatorname{dim} \tau=d+1} H_{(i)}^{i}\left(K_{\tau}^{R} ; k\right) .
$$

We conclude the first part using the equality $K_{\tau}^{R}=\cap_{a_{j} \in \tau} K_{a_{j}}^{R}$ and Proposition 4.8

If $X$ is an isolated singularity then we also have $E_{1}^{p, q}=0$ for $p \geq d+2$. Thus $E_{2}^{d+1, q}=E_{\infty}^{d+1, q}=H_{(i)}^{q}\left(K_{\sigma}^{R} ; k\right)$ for $q \geq i+1$, which finishes the proof.

Corollary 4.10. Since toric varieties are normal and thus smooth in codimension 1, we obtain that $T_{(i)}^{1}(-R)$ equals the cohomology group of the complex

$$
\bar{C}_{(i)}^{i}\left(M_{k} ; k\right) \rightarrow \oplus_{j} \bar{C}_{(i)}^{i}\left(\operatorname{Span}_{k} E_{j}^{R} ; k\right) \rightarrow \oplus_{\left\langle a_{j}, a_{k}\right\rangle<\sigma} \bar{C}_{(i)}^{i}\left(\operatorname{Span}_{k} E_{j k}^{R} ; k\right) .
$$

4.4. Toric surfaces. We want to obtain the dimension of $k$-vector spaces $T_{(i)}^{1,-R}(A)$, for all $i \in \mathbb{N}$, in the case when $A$ is a two-dimensional cyclic quotient singularity (a two-dimensional affine toric variety). Let $X(n, q)$ denote the quotient by the $\mathbb{Z} / n \mathbb{Z}$-action $\xi \rightarrow\left(\begin{array}{cc}\xi & 0 \\ 0 & \xi^{q}\end{array}\right),(\xi=\sqrt[n]{1}) . \quad X(n, q)$ is given by the cone $\sigma=\left\langle a_{1}, a_{2}\right\rangle=\langle(1,0),(-q, n)\rangle$. We can develop $\frac{n}{n-q}$ into a continued fraction $\left[b_{1} ; b_{2}, \ldots, b_{r}\right], b_{i} \geq 2$. Then $E$ is given as the set $E=\left\{w^{0}, \ldots, w^{r+1}\right\}$, with elements $w^{i} \in \mathbb{Z}^{2}$ and

(1) $w^{0}=(0,1), w^{1}=(1,1), w^{r+1}=(n, q)$,

(2) $w^{i-1}+w^{i+1}=b_{i} \cdot w^{i}(\mathrm{i}=1, \ldots, \mathrm{r})$.

Now we compute $T_{(i)}^{1,-R}(A)$ for toric surfaces $A=A(n, q):=k\left[\left\langle w^{0}, w^{r+1}\right\rangle \cap M\right]$.

Proposition 4.11. For $i>2$ we have $\operatorname{dim} T_{(i)}^{1,-R}(A)=0$. Otherwise we have

$$
\begin{gathered}
\operatorname{dim}_{k} T_{(i)}^{1,-R}(A)= \\
\operatorname{dim}_{k} \bar{C}_{(i)}^{i}\left(\operatorname{Span}_{k} E_{1}^{R} ; k\right)+\operatorname{dim}_{k} \bar{C}_{(i)}^{i}\left(\operatorname{Span}_{k} E_{2}^{R} ; k\right)-\operatorname{dim}_{k} \bar{C}_{(i)}^{i}\left(\operatorname{Span}_{k} E_{12}^{R} ; k\right)-c_{i},
\end{gathered}
$$

where

$$
c_{i}:= \begin{cases}2=\operatorname{dim}_{k} \bar{C}_{(1)}^{1}\left(M_{k} ; k\right) & \text { if } i=1 \\ 1=\operatorname{dim}_{k} \bar{C}_{(2)}^{2}\left(M_{k} ; k\right) & \text { if } i=2\end{cases}
$$

Proof. Follows immediately from (6), where in this case the last map is surjective.

Corollary 4.12. For $T_{(1)}^{1}(A)$ we obtain the same results as Pinkham [27]. Focusing on $T_{(2)}^{1,-R}(A)$, there are four different cases for the multidegree $R \in M \cong \mathbb{Z}^{2}$ :

- $R=w^{1}$ (or analogously $R=w^{r}$ ). We obtain $E_{1}=\left\{w^{0}\right\}$ and $E_{2}=$ $\left\{w^{2}, \ldots, w^{r+1}\right\}$. We have

$$
\operatorname{dim}_{k} \bar{C}_{(2)}^{2}\left(\operatorname{Span}_{k} E_{1}^{R} ; k\right)=\operatorname{dim}_{k} \bar{C}_{(2)}^{2}\left(\operatorname{Span}_{k} E_{12}^{R} ; k\right)=0
$$

and thus Proposition 4.11 yields $T_{(2)}^{1,-R}(A)=0$.

- $R=w^{i}(2 \leq i \leq r-1)$. We obtain $E_{1}=\left\{w^{0}, \ldots, w^{i-1}\right\}$ and $E_{2}=$ $\left\{w^{i+1}, \ldots, w^{r+1}\right\}$. We have $\operatorname{dim}_{k} \bar{C}_{(2)}^{2}\left(\operatorname{Span}_{k} E_{12}^{R} ; k\right)=0$,

$$
\operatorname{dim}_{k} \bar{C}_{(2)}^{2}\left(\operatorname{Span}_{k} E_{1}^{R} ; k\right)=\operatorname{dim}_{k} \bar{C}_{(2)}^{2}\left(\operatorname{Span}_{k} E_{2}^{R} ; k\right)=1
$$

and Proposition 4.11 yields $\operatorname{dim}_{k} T_{(2)}^{1,-R}(A)=1$. 
- $R=l \cdot w^{i}\left(1 \leq i \leq r, 2 \leq l \leq b_{i}\right.$ for $r \geq 2$, or $i=1,2 \leq l \leq b_{1}$ for $r=1)$. We obtain $E_{1}=\left\{w^{0}, \ldots, w^{i}\right\}$ and $E_{2}=\left\{w^{i}, \ldots, w^{r+1}\right\}$. We have $\operatorname{dim}_{k} \bar{C}_{(2)}^{2}\left(\operatorname{Span}_{k} E_{12}^{R} ; k\right)=0$,

$$
\operatorname{dim}_{k} \bar{C}_{(2)}^{2}\left(\operatorname{Span}_{k} E_{1}^{R} ; k\right)=\operatorname{dim}_{k} \bar{C}_{(2)}^{2}\left(\operatorname{Span}_{k} E_{2}^{R} ; k\right)=1
$$

and thus Proposition 4.11 yields $\operatorname{dim}_{k} T_{(2)}^{1,-R}=1$.

- For the remaining $R \in M$, either $E_{1} \subset E_{2}$ or $E_{2} \subset E_{1}$ or $\#\left(E_{1} \cap E_{2}\right) \geq 2$. In these cases it holds that either $\operatorname{dim}_{k} \bar{C}_{(2)}^{2}\left(\operatorname{Span}_{k} E_{i}^{R} ; k\right)=0$ for some $i$, or we have $\operatorname{dim}_{k} \bar{C}_{(2)}^{2}\left(\operatorname{Span}_{k} E_{12}^{R} ; k\right) \neq 0$. Thus in all these cases Proposition 4.11 yields $\operatorname{dim}_{k} T_{(2)}^{1,-R}(A)=0$.

The following example shows that in the case of Gorenstein toric surfaces $\left(A_{n^{-}}\right.$ singularities) the computations in this section agree with the computations in the previous section.

Example 3. Let $A=A(n+1, n)$ be a Gorenstein toric surface, given by the polynomial $f(x, y, z)=x y-z^{n+1}$ in $\mathbb{A}^{3}$. From Proposition 3.3 we know that $\mathrm{HH}^{3}(A) \cong A /\left(\frac{\partial f}{\partial x}, \frac{\partial f}{\partial y}, \frac{\partial f}{\partial z}\right)$, which has dimension equal to $n$ (Milnor number of the hypersurface). From Lemma 3.2 we have $\operatorname{HH}^{3}(A) \cong \oplus_{i=0}^{2} \operatorname{Ext}^{i}\left(\Omega_{A \mid k}^{3-i}, A\right)$ and since $\operatorname{Ext}_{A}^{2}\left(\Omega_{A \mid k}, A\right)=\operatorname{Hom}\left(\Omega_{A \mid k}^{3}, A\right)=0$, we see that $\operatorname{HH}^{3}(A) \cong T_{(2)}^{1}(A) \cong$ $\operatorname{Ext}^{1}\left(\Omega_{A \mid k}^{2}, A\right)$ and thus $\operatorname{dim}_{k} T_{(2)}^{1}(A)=n$. Using Corollary 4.12 we can be even more precise: the cone for $A$ is given by $\sigma=\langle(1,0),(-n, n+1)\rangle$. Its continued fraction has $r=1, b_{1}=n+1$ and thus we have $\operatorname{dim}_{k} T_{(2)}^{1,-R}(A)=1$ for the degrees $R=(2,2), \ldots,(n+1, n+1)$ and $\operatorname{dim}_{k} T_{(2)}^{1,-R}(A)=0$ for the other degrees.

4.5. Higher dimensions. Let the cone $\sigma=\left\langle a_{1}, \ldots, a_{N}\right\rangle$ represent an $n$-dimensional toric variety $X_{\sigma}=\operatorname{Spec}(A), n \geq 3$. For $R \in M$ we define the affine space

$$
\mathbb{A}(R):=\left\{a \in N_{\mathbb{R}} \mid\langle a, R\rangle=1\right\} \subset N_{\mathbb{R}}
$$

and consider the polyhedron $Q(R):=\sigma \cap \mathbb{A}(R) \subset \mathbb{A}(R)$. Vertices of $Q(R)$ are $\bar{a}_{j}:=a_{j} /\left\langle a_{j}, R\right\rangle$, for all $j$ satisfying $\left\langle a_{j}, R\right\rangle \geq 1$. We denote $T_{(i)}^{1}(-R):=T_{(i)}^{1,-R}(A)$.

Altmann [2], 3] relates the computation of $T_{(1)}^{1}(-R)$ with the convex geometry of $Q(R)$ (using Minkowski summands of $Q(R)$ ). We will develop another approach that will also allow us to compute $T_{(i)}^{1}(-R)$ for $i>1$. At the end we will obtain explicit formulas for 3-dimensional toric varieties (see Proposition 4.14). As far as we know the techniques that we use to obtain this calculations are new even in the case $i=1$. In this subsection we also obtain a formula for $T_{(i)}^{1}(-R)$ for affine cones over smooth toric Fano varieties in arbitrary dimension (see Theorem 4.18).

The following lemma will be useful.

Lemma 4.13. Let $Y$ be a toric surface given by $\sigma=\left\langle a_{1}, a_{2}\right\rangle \subset N_{\mathbb{R}} \cong \mathbb{R}^{2}$. We have $\operatorname{dim}_{k} \operatorname{Span}_{k} E_{12}^{R}=\max \left\{0, W_{1}(R)+W_{2}(R)-2-\operatorname{dim}_{k} T_{(1)}^{1,-R}(Y)\right\}$, where

$$
W_{j}(R):=\left\{\begin{array}{cc}
2 & \text { if }\left\langle a_{j}, R\right\rangle>1 \\
1 & \text { if }\left\langle a_{j}, R\right\rangle=1 \\
0 & \text { if }\left\langle a_{j}, R\right\rangle \leq 0
\end{array}\right.
$$

Proof. It follows immediately by Proposition 4.11 
Let $d_{j k}:=\overline{\bar{a}_{j} \bar{a}_{k}}$ denote the compact edges of $Q(R)$ (for $\left\langle a_{j}, a_{k}\right\rangle \leq \sigma,\left\langle a_{j}, R\right\rangle \geq 1$, $\left.\left\langle a_{k}, R\right\rangle \geq 1\right)$. We denote the lattice $N \cap \operatorname{Span}_{k}\left\langle a_{j}, a_{k}\right\rangle$ by $\bar{N}_{j k}$ and its dual with $\bar{M}_{j k}$. Let $\bar{R}_{j k}$ denote the projection of $R$ to $\bar{M}_{j k}$.

Proposition 4.14. If the compact part of $Q(R)$ lies in a two-dimensional affine space we have

$$
\operatorname{dim}_{k} T_{(i)}^{1}(-R)=\max \left\{0, \sum_{j=1}^{N} V_{j}^{i}(R)-\sum_{d_{j k} \in Q(R)} Q_{j k}^{i}(R)-\left(\begin{array}{c}
n \\
i
\end{array}\right)+s_{Q(R)}^{i}\right\},
$$

where

$$
\begin{aligned}
& V_{j}^{i}(R):= \begin{cases}\left(\begin{array}{c}
n \\
i
\end{array}\right) & \text { if }\left\langle a_{j}, R\right\rangle>1 \\
\left(\begin{array}{c}
n-1 \\
i
\end{array}\right) & \text { if }\left\langle a_{j}, R\right\rangle=1 \\
0 & \text { if }\left\langle a_{j}, R\right\rangle \leq 0,\end{cases} \\
& Q_{j k}^{i}(R):= \begin{cases}\left({ }^{W_{j}(R)+W_{k}(R)+n-4-\operatorname{dim}_{k} T_{\left\langle a_{j}, a_{k}\right\rangle}^{1}\left(-\bar{R}_{j k}\right)}\right) & \text { if }\left\langle a_{j}, R\right\rangle,\left\langle a_{k}, R\right\rangle \neq 0 \\
0 & \text { otherwise }\end{cases} \\
& s_{Q(R)}^{i}:= \begin{cases}\operatorname{dim}_{k} \wedge^{i}\left(\bigcap_{d_{j k} \in Q(R)} \operatorname{Span}_{k} E_{j k}^{R}\right) & \text { if } Q(R) \text { is compact } \\
0 & \text { otherwise }\end{cases}
\end{aligned}
$$

Proof. From Theorem 4.9 we know that $T_{(i)}^{1}(-R)$ is the cohomology group of the complex

$$
\bar{C}_{(i)}^{i}\left(M_{k} ; k\right) \rightarrow \oplus_{j} \bar{C}_{(i)}^{i}\left(\operatorname{Span}_{k} E_{j}^{R} ; k\right) \rightarrow \oplus_{\left\langle a_{j}, a_{k}\right\rangle \leq \sigma} \bar{C}_{(i)}^{i}\left(\operatorname{Span}_{k}\left(E_{j k}^{R}\right) ; k\right) .
$$

Let $f:=\left(f_{1}, \ldots, f_{N}\right) \in \oplus_{j} \bar{C}_{(i)}^{i}\left(\operatorname{Span}_{k} E_{j}^{R}\right)$. We see that $V_{j}^{i}(R)=\operatorname{dim}_{k}\left(\wedge^{i} \operatorname{Span}_{k} E_{j}^{R}\right)$. Assume now that $\operatorname{Span}_{k} E_{j}^{R}, \operatorname{Span}_{k} E_{k}^{R} \neq \emptyset$, otherwise we have $\operatorname{Span}_{k} E_{j k}^{R}=\emptyset$. We can easily verify that $Q_{j k}^{i}(R)=\operatorname{dim}_{k}\left(\wedge^{i} \operatorname{Span}_{k} E_{j k}^{R}\right)$ :

we have $\operatorname{dim}_{k}\left(\operatorname{Span}_{k} E_{j k}^{R}\right)=n-2+\operatorname{dim}_{k}\left(\operatorname{Span}_{k} \bar{E}_{j k}^{\bar{R}_{j k}}\right)$, where $\bar{E}_{j k}$ is the generating set of $\left\langle a_{j}, a_{k}\right\rangle^{\vee} \cap \bar{M}_{j k}$. From Lemma 4.13 we know that $\operatorname{dim}_{k}\left(\operatorname{Span}_{k} \bar{E}_{j k}^{\bar{R}}\right)=$ $\max \left\{0, W_{j}(R)+W_{k}(R)-2-\operatorname{dim}_{k} T_{\left\langle a_{j}, a_{k}\right\rangle}^{1}\left(-\bar{R}_{j k}\right)\right\}$. Thus we have

$$
\operatorname{dim}_{k} T_{(i)}^{1}(-R)=\max \left\{0, \sum_{j=1}^{N} V_{j}^{i}(R)-\sum_{d_{j k}} Q_{j k}^{i}(R)-\left(\begin{array}{c}
n \\
i
\end{array}\right)+s^{i}\right\},
$$

where $s^{i}$ equals the dimension of the domain of restrictions (that we get with restricting $f_{j}=f_{k}$ on $\operatorname{Span}_{k} E_{j k}$ ) that repeats. We can easily verify that $s^{i}=$ $s_{Q(R)}^{i}$.

Using Proposition 4.14 we can easily compute $T_{(i)}^{1}(-R)$ for three-dimensional affine toric varieties. From straightforward computation of the formula in Proposition 4.14 we obtain the following corollary.

Corollary 4.15. Let $X$ be an isolated 3-dimensional toric singularity. Without loss of generality we can assume that generators $a_{1}, \ldots, a_{N}$ are arranged in a cycle. 
We have the following formulas:

$$
\begin{aligned}
& \operatorname{dim}_{k} T_{(1)}^{1}(-R)= \\
& = \begin{cases}\max \left\{0, \#\left\{\bar{a}_{j} \mid \bar{a}_{j} \in N, \text { i.e. }\left\langle a_{j}, R\right\rangle=1\right\}-3\right\} & \text { if } R>0 \\
\max \left\{0, \#\left\{\bar{a}_{j} \mid \bar{a}_{j} \in N, \text { not contained in a non-compact edge }\right\}\right. & \text { if } R \ngtr 0,\end{cases} \\
& \operatorname{dim}_{k} T_{(2)}^{1}(-R)= \begin{cases}\max \left\{0, \#\left\{\bar{a}_{j} \mid \bar{a}^{j} \in N\right\}+C(R)-3\right\} & \text { if } R>0 \\
\max \left\{0, \#\left\{\bar{a}_{j} \mid \bar{a}^{j} \in N\right\}+C(R)-2\right\} & \text { if } R \ngtr 0,\end{cases} \\
& \operatorname{dim}_{k} T_{(3)}^{1}(-R)=\max \{0, C(R)-1\} \\
& \operatorname{dim}_{k} T_{(i)}^{1}(-R)=0 \text { for } i \geq 4,
\end{aligned}
$$

where $C(R):=\#\left\{\right.$ chambers with $\left.\left\langle a_{j}, R\right\rangle>1\right\}$ and a chamber with $\left\langle a_{j}, R\right\rangle>1$ means $\left\langle a_{j}, R\right\rangle>1$ for $j=j_{0}, j_{0}+1, \ldots, j_{0}+k$ for some $j_{0}, k \in \mathbb{N}$ and $\left\langle a_{j}, R\right\rangle \ngtr 1$ for $j=j_{0}-1$ and $j=j_{0}+k+1$.

Proof. We use Theorem 4.14 with $n=3$. We also have $T_{\left\langle a_{j}, a_{j+1}\right\rangle}^{1}\left(-\bar{R}_{j, j+1}\right)=0$ for all $j$ since $X$ is smooth in codimension 2 . Let $m_{1}$ be a number of $a_{j}$ with $\left\langle a_{j}, R\right\rangle=1$ (i.e. $m_{1}$ is the number of lattice vertices of the polytope $Q(R)$ ) and $m_{2}$ be a number of vertices $a_{j}$ with $\left\langle a_{j}, R\right\rangle>1$.

If $R>0$ we have $N=m_{1}+m_{2}$ and thus we can easily compute that

$$
s_{Q(R)}^{i}=\operatorname{dim}_{k} \wedge^{i} \bigcap_{j} \operatorname{Span}_{k} E_{j, j+1}^{R}=\left(\begin{array}{c}
\max \left\{0,3-m_{1}\right\} \\
i
\end{array}\right) .
$$

For $i=1$ we have $\sum_{j=1}^{N} V_{j}^{1}(R)=3 m_{2}+2 m_{1}, \sum_{j=1}^{N} W_{j}(R)=2 m_{1}+m_{2}$ and thus $\sum_{d_{j}} Q_{j, j+1}^{1}(R)=2 \sum_{j=1}^{N}\left(W_{j}(R)\right)-N=4 m_{2}+2 m_{1}-m_{1}-m_{2}=3 m_{2}+m_{1}$. Thus we see that $T_{(1)}^{1}(-R)=\max \left\{0, m_{1}-3\right\}$.

For $i=2$ we have

$$
Q_{j, j+1}^{2}(R)= \begin{cases}3 & \text { if } V_{j}^{2}(R)=V_{j+1}^{2}(R)=3 \\ 1 & \text { if } V_{j}^{2}(R)=2, V_{j+1}^{2}(R)=3 \text { or } V_{j}^{2}(R)=3, V_{j+1}^{2}(R)=2 \\ 0 & \text { otherwise }\end{cases}
$$

and thus

$$
V_{j}^{2}(R)-Q_{j, j+1}^{2}(R)= \begin{cases}1 & \text { if }\left\langle a_{j}, R\right\rangle=1 \text { and }\left\langle a_{j+1}, R\right\rangle=1 \\ 0 & \text { if }\left\langle a_{j}, R\right\rangle=1 \text { and }\left\langle a_{j+1}, R\right\rangle=2 \\ 2 & \text { if }\left\langle a_{j}, R\right\rangle=2 \text { and }\left\langle a_{j+1}, R\right\rangle=1 \\ 0 & \text { if }\left\langle a_{j}, R\right\rangle=2 \text { and }\left\langle a_{j+1}, R\right\rangle=2 \\ 0 & \text { otherwise }\end{cases}
$$

from which we easily obtain the formula that we want. For $i=3$ we have $\sum_{j=1}^{N} V_{j}^{3}(R)=m_{2}$,

$$
Q_{j, j+1}^{3}(R)= \begin{cases}1 & \text { if } V_{j}^{3}(R)=V_{j+1}^{3}(R)=3 \\ 0 & \text { otherwise }\end{cases}
$$

and the formula follows.

If $R \ngtr 0$ we do not have any compact 2 -faces in $Q(R)$. The only nontrivial case is when we have two vertices that lie on the unbounded edges. We skip this computations since they are similar as in the case $R>0$. 
Remark 3. When $Q(R)$ is not contained in a two-dimensional affine space, we can still follow the proof of Proposition 4.14 and we obtain that

$$
\operatorname{dim}_{k} T_{(i)}^{1}(-R) \geq \sum_{j=1}^{N} V_{j}^{i}(R)-\sum_{d_{j k} \in Q(R)} Q_{j k}^{i}(R)-\left(\begin{array}{c}
n \\
i
\end{array}\right) .
$$

The cycles in $Q(R)$ give us some repetitions on the restrictions $\left(f_{j}=f_{k}\right.$ on $\left.\operatorname{Span}_{k} E_{j k}^{R}\right)$ and thus it is hard to obtain a formula for $\operatorname{dim}_{k} T_{(i)}^{1}(-R)$ in higher dimensions. For every tree $T$ in $Q(R)$ we obtain also upper bounds:

$$
\operatorname{dim}_{k} T_{(i)}^{1}(-R) \leq \sum_{j=1}^{N} V_{j}^{i}(R)-\sum_{d_{j k} \in T} Q_{j k}^{i}(R)-\left(\begin{array}{c}
n \\
i
\end{array}\right),
$$

since no cycles appear in $T$.

We focus now on higher dimensional toric varieties. We will analyse the case of $\mathbb{Q}$-Gorenstein toric varieties that are smooth in codimension two.

Lemma 4.16. Let $Y$ be a $\mathbb{Q}$-Gorenstein variety which is smooth in codimension two. If $R \in M$ is a degree such that $\left\langle a_{j}, R\right\rangle \geq 2$ for some $j \in\{1, \ldots, N\}$, then $T_{(i)}^{1}(-R)=0$ for all $i \geq 1$.

Proof. The hyperplane $H:=\left\{a \in N_{\mathbb{R}} \mid\left\langle a, g R-R^{*}\right\rangle=0\right\}$ subdivides the set of generators of $\sigma: H_{<0}^{R}:=\left\{a_{j} \mid\left\langle a_{j}, R\right\rangle \leq 0\right\}, H_{1}^{R}=\left\{a_{j} \mid\left\langle a_{j}, R\right\rangle=1\right\}$ and $H_{\geq 2}^{R}=\left\{a_{j} \mid\left\langle a_{j}, R\right\rangle \geq 2\right\}$. We fix a vertex $\bar{a}_{j_{0}}$ of $Q(R)$ with $\left\langle a_{j_{0}}, R\right\rangle \geq 2$. Skipping some of the edges, we can arrange $Q(R)$ into a tree $T$ with the main vertex $\bar{a}_{j_{0}}$, the set of leaves equal to $H_{1}^{R}$ and the set of inner vertices equal to $H_{\geq 2}^{R} \backslash \bar{a}_{j_{0}}$. From the equation (8) we see that $\operatorname{dim}_{k} T_{(i)}^{1}(-R) \leq \sum_{j=1}^{N} V_{j}^{i}(R)-\sum_{d_{j k} \in T} Q_{j k}^{i}(R)-\left(\begin{array}{c}n \\ i\end{array}\right)$ and we can easily verify that this is $\leq 0$.

Deformation theory of affine varieties is closely related to the Hodge theory of smooth projective varieties. We will use the following recent result.

Theorem 4.17. Let $X=\operatorname{Spec}(A)$ be an affine cone over a projective variety $Y$. On $T_{(i)}^{q}(A)$ we have a natural $\mathbb{Z}$ grading and if $Y$ is arithmetically Cohen-Macaulay and $\omega_{Y} \cong \mathcal{O}_{Y}(m)$, then

$$
T_{(i)}^{q}(A)_{m}= \begin{cases}H_{p r i m}^{n-i, q}(Y) & \text { if } i>q \\ H_{p r i m}^{n-q-1, i}(Y) & \text { if } i \leq q,\end{cases}
$$

where $T_{(i)}^{q}(A)_{m}$ denotes the degree $m \in \mathbb{Z}$ elements of $T_{(i)}^{q}(A)$ and $H_{\text {prim }}^{p, q}(Y)$ is the primitive cohomology, namely the kernel of the Lefschetz maps

$$
H^{p, q}(Y) \rightarrow H^{p+1, q+1}(Y) .
$$

Proof. See [12, Corollary 3.14].

We will apply Theorem 4.17 to the case of Fano toric varieties, where reflexive polytopes come into the play.

Definition 12. A full dimensional lattice polytope $P \subset M_{\mathbb{R}}$ is called reflexive if $0 \in \operatorname{int}(P)$ and, moreover, its dual

$$
P^{\vee}:=\left\{a \in N_{\mathbb{R}} \mid\langle a, P\rangle \geq-1\right\}
$$


is also a lattice polytope. Here the expression $\langle a, P\rangle$ means the minimum over the set $\{\langle a, r\rangle \mid r \in P\}$.

Reflexive polytopes lead to interesting toric varieties that are important for mirror symmetry. There is a one-to-one correspondence between Gorenstein toric Fano varieties and reflexive polytopes (see [11, Theorem 8.3.4]).

If $X$ is a Gorenstein affine toric variety given by $\sigma=\operatorname{Cone}(P)$, where $P$ is a reflexive polytope, then $X$ is an affine cone over a smooth Fano toric variety $Y$, embedded in some $\mathbb{P}^{n}$ by the anticanonical line bundle.

Theorem 4.18. Let $X=\operatorname{Spec}(A)$ be an $n$-dimensional affine cone over a smooth toric Fano variety $Y(n \geq 3)$. Then $T_{(i)}^{1}(A)=0$ for $n \geq 4$ and $i=2, \ldots, n-2$. Moreover, $\operatorname{dim}_{k} T_{(n-1)}^{1}(A)=N-n$ and $T_{(k)}^{1}(A)=0$ for $k \geq n \geq 3$. Furthermore, $\operatorname{dim}_{k} T_{(1)}^{1}(A)=N-3$ for $n=3$ and $T_{(1)}^{1}(A)=0$ for $n>3$.

Proof. It holds that $H^{p, q}(Y)=0$ for $p \neq q$ (see e.g. [7]) and thus also $H_{\text {prim }}^{p, q}(Y)=0$. By Theorem 4.17 we have $T_{(i)}^{1}(A)_{-1}=0$ for $n \geq 4$ and $i=2, \ldots, n-2$. Following the proof of Lemma 4.16, we see that if $R \neq R^{*}=(\underline{0}, 1)$ we have the following options:

(1) there exists $a_{j}$, such that $\left\langle a_{j}, R\right\rangle \geq 2$, which implies that $T_{(i)}^{1,-R}(A)=0$ for all $i \geq 1$ by Lemma 4.16

(2) $H_{\geq 2}^{R}=0$ and $H_{1}^{R}=\left\{a_{j} \in F\right\}$ for a facet $F$. There exists $s \in M$ such that $\left\langle s, a_{j}\right\rangle=0$ for all $a_{j} \in F$. If $T_{(i)}^{1,-R}(A) \neq 0$ for some $i$, then $\operatorname{dim}_{k} T_{(i)}^{1,-R+\alpha s}(A) \neq 0$ for infinitely many $\alpha \in \mathbb{Z}$. Thus $\operatorname{dim}_{k} T_{(i)}^{1}(A)=\infty$, which is a contradiction since $T_{(i)}^{1}(A)$ is supported on the singular locus and $A$ is an isolated singularity. Thus $T_{(i)}^{1,-R}(A)=0$ for all $i \geq 1$.

(3) $H_{\geq 2}^{R}=H_{1}^{R}=0$, which trivially implies that $T_{(i)}^{1,-R}(A)=0$.

Now we focus in the case $i=n-1$. Above we saw that $T_{(n-1)}^{1,-R}(A)=0$ if $R \neq R^{*}$. The inequality (7) is in the case $R=R^{*}, i=n-1$ an equality since no restrictions repeat and thus we obtain

$$
\operatorname{dim}_{k} T_{(n-1)}^{1,-R^{*}}(A)=\max \left\{0, \sum_{j=1}^{N} V_{j}^{n-1}\left(R^{*}\right)-\sum_{d_{j k} \in Q\left(R^{*}\right)} Q_{j k}^{n-1}\left(R^{*}\right)-\left(\begin{array}{c}
n \\
n-1
\end{array}\right)\right\} .
$$

Since $V_{j}^{n-1}\left(R^{*}\right)=\left(\begin{array}{l}n-1 \\ n-1\end{array}\right)=1$ and $Q_{j k}^{n-1}\left(R^{*}\right)=\left(\begin{array}{l}n-2 \\ n-1\end{array}\right)=0$ we obtain $T_{(n-1)}^{1,-R^{*}}(A)=$ $N-n$. With the same procedure we immediately see that $T_{(k)}^{1}(A)=0$ for $k \geq n$. Finally we focus on the case $i=1$. With the same computations as above we see that $\operatorname{dim}_{k} T_{(1)}^{1}(A)=0$ if $n>3$. If $n=3$, then $\operatorname{dim}_{k} T_{(1)}^{1}(A)_{-1}=\operatorname{dim}_{k} T_{(1)}^{1}(A)$ as above and $T_{(1)}^{1}(A)=H_{\text {prim }}^{1,1}(Y)$ by Theorem 4.17. We have $\operatorname{dim}_{k} H_{\text {prim }}^{1,1}(Y)=N-3$ by [11, Theorem 9.4.11] and thus we conclude the proof.

Remark 4. From Theorem 4.18 and Theorem 4.17 it follows that

$$
\operatorname{dim}_{k} H_{\text {prim }}^{1,1}(Y)=N-n=\operatorname{rk}(\operatorname{Pic}(Y))-1 .
$$

For $i=n-2$ we can generalize Theorem 4.18 to the following:

Proposition 4.19. Let $X=\operatorname{Spec}(A)$ be $n$-dimensional $\mathbb{Q}$-Gorenstein variety given by $\sigma=\operatorname{Cone}(P)$, where $P$ is a simplicial polytope. Then $T_{(n-2)}^{1}(A)=0$. 
Proof. The only non-clear part is when $X$ is Gorenstein and we consider the degree $R=R^{*}$. Again following the proof of Proposition 4.14 we see that

$$
\operatorname{dim}_{k} T_{(n-2)}^{1,-R^{*}}(A)=\max \left\{0, \sum_{j=1}^{N} V_{j}^{n-2}\left(R^{*}\right)-\sum_{d_{j k} \in Q\left(R^{*}\right)} Q_{j k}^{n-2}\left(R^{*}\right)-\left(\begin{array}{c}
n \\
n-2
\end{array}\right)\right\},
$$

since no restrictions repeat. Let $e$ denote the number of edges in $Q\left(R^{*}\right)$. Since $V_{j}^{n-2}\left(R^{*}\right)=\left(\begin{array}{l}n-1 \\ n-2\end{array}\right)=n-1$ and $Q_{j k}^{n-2}\left(R^{*}\right)=\left(\begin{array}{c}n-2 \\ n-2\end{array}\right)=1$, we obtain $\operatorname{dim}_{k} T_{(i)}^{1}\left(-R^{*}\right)=$ $\max \{0, N(n-1)-e-n(n-1) / 2\}$. For simplicial polytopes it holds that $e \geq$ $N(n-1)-n(n-1) / 2$ by the lower bound conjecture proved in [6] and thus $\operatorname{dim}_{k} T_{(i)}^{1}\left(-R^{*}\right)=0$

Remark 5. For $i=1$ we can generalize Theorem 4.18 to the following: $\mathbb{Q}$ Gorenstein toric varieties that are smooth in codimension 2 and $\mathbb{Q}$-factorial (or equivalently simplicial) in codimension 3 are globally rigid (see [30 or [1] for the affine case).

\section{Deformation Quantization of AFFine toric Varieties}

In this section we prove that every Poisson structure on an affine toric variety can be quantized. We will use the Maurer-Cartan formalism, Kontsevich's formality theorem (or more precisely its corollary 5.3) and the GIT quotient construction for an affine toric variety $\operatorname{Spec}(A)$ without torus factors: we can write $\operatorname{Spec}(A)=$ $\mathbb{A}^{N} / / G$ for some group $G$. This construction works over an algebraically closed field $k$ of characteristic 0 . The proof of deformation quantization works also in the case of affine toric varieties with torus factors.

Definition 13. Let $\mathfrak{g}$ be a differential graded Lie algebra. The Maurer-Cartan equation is

$$
d \xi+\frac{1}{2}[\xi, \xi]=0, \quad \xi \in \mathfrak{g}^{1},
$$

where $\mathfrak{g}^{1}$ denotes the set of degree 1 elements in $\mathfrak{g}$. A solution of this equation is called a Maurer-Cartan (an MC) element.

Lemma 5.1. One parameter formal deformations $(A[[\hbar]], *)$ of $A$ are in bijection with $M C$ elements of a dgla $\mathfrak{g}:=(\hbar C \bullet(A)[1])[[\hbar]]$.

Proof. See [29, Proposition 4.3.1].

Theorem 5.2 (Formality theorem [20], 14]). Let $X=\operatorname{Spec}(A)$ be a smooth affine variety. There exists an $L_{\infty}$-quasi-isomorphism between the Hochschild dgla $C^{\bullet}(A)[1]$ and the formal dgla $H^{\bullet}(A)[1]$ (i.e. the graded Lie algebra $H^{\bullet}(A)[1]$ with trivial differential).

Corollary 5.3. Every Poisson structure $\pi$ on a smooth affine variety $\operatorname{Spec}(A)$ can be quantized.

Now we focus to the case of (singular) toric varieties. Using the lattice grading the Gerstenhaber bracket can be simplified as follows.

Lemma 5.4. Let $A=k[\Lambda], f\left(x^{\lambda_{1}}, \ldots, x^{\lambda_{m}}\right)=\sum_{i=0}^{p} f_{i}\left(\lambda_{1}, \ldots, \lambda_{m}\right) x^{-R_{i}+\lambda_{1}+\cdots+\lambda_{m}} \in$ $C^{m}(A)$ and $g\left(x^{\lambda_{1}}, \ldots, x^{\lambda_{n}}\right)=\sum_{j=0}^{r} g_{j}\left(\lambda_{1}, \ldots, \lambda_{n}\right) x^{-S_{j}+\lambda_{1}+\cdots \lambda_{n}} \in C^{n}(A)$, where $f_{i} \in$ 
$C^{m}\left(\Lambda, \Lambda \backslash\left(R_{i}+\Lambda\right) ; k\right)$, for $i=0, . ., p$ and $g_{j} \in C^{n}\left(\Lambda, \Lambda \backslash\left(S_{j}+\Lambda\right) ; k\right)$ for $j=0, \ldots, r$. Then

$$
[f, g]\left(x^{\lambda_{1}}, \ldots, x^{\lambda_{m+n-1}}\right)=\sum_{i, j}\left[f_{i}, g_{j}\right] x^{-R_{i}-S_{j}+\lambda_{1}+\cdots \lambda_{m+n-1}},
$$

where

$$
\left[f_{i}, g_{j}\right]:=f_{i} \circ g_{j}-(-1)^{(m+1)(n+1)} g_{j} \circ f_{i} \in C^{m+n-1}\left(\Lambda, \Lambda \backslash\left(R_{i}+S_{j}+\Lambda\right) ; k\right),
$$

where $f_{i} \circ g_{j}\left(\lambda_{1}, \ldots, \lambda_{m+n-1}\right):=$

$\sum_{u=1}^{m} k_{u}^{n} \cdot f_{i}\left(\lambda_{1}, \ldots, \lambda_{u-1},-S_{j}+\lambda_{u}+\cdots+\lambda_{u+n-1}, \lambda_{u+n}, \ldots, \lambda_{m+n-1}\right) g_{j}\left(\lambda_{u}, \ldots, \lambda_{u+n-1}\right)$, where $k_{u}^{n}=(-1)^{(u-1)(n+1)}$.

Proof. It follows from the isomorphism in Lemma 4.1 .

Proposition 5.5. Every Poisson structure $p$ on an affine toric variety $\operatorname{Spec}(k[\Lambda])$ is of the form

$$
p\left(x^{\lambda_{1}}, x^{\lambda_{2}}\right)=\sum_{i=0}^{d} f_{i}\left(\lambda_{1}, \lambda_{2}\right) x^{R_{i}+\lambda_{1}+\lambda_{2}},
$$

where $f_{i} \in \bar{C}_{(2)}^{2}\left(\Lambda, \Lambda \backslash\left(-R_{i}+\Lambda\right) ; k\right), R_{i} \in M$. We call $f_{i}\left(\lambda_{1}, \lambda_{2}\right) x^{R_{i}+\lambda_{1}+\lambda_{2}}$ the Poisson structure of degree $R_{i}$ and we call $p$ a Poisson structure of index $\left(R_{0}, \ldots, R_{d}\right)$.

Proof. A Poisson structure $p$ is an element of $H_{(2)}^{2}(k[\Lambda])$ such that $e_{3}(3)[p, p]=0$. From Proposition 4.2 and 4.7 we know that

$$
H_{(n)}^{n, R}(k[\Lambda])=H_{(n)}^{n}(\Lambda, \Lambda \backslash(-R+\Lambda) ; k)=\bar{C}_{(n)}^{n}(\Lambda, \Lambda \backslash(-R+\Lambda) ; k),
$$

thus $p$ is of the form (9), and $e_{3}(3)[p, p]=0$ gives us additional restrictions on $f_{i}$, $i=0, . ., d$.

Example 4. For every hypersurface given by the polynomial $g(x, y, z)$ in $\mathbb{A}^{3}$, we can define a Poisson structure $\pi_{g}$ on the quotient $k[x, y, z] / g$, namely:

$$
\pi_{g}:=\partial_{x}(g) \partial_{y} \wedge \partial_{z}+\partial_{y}(g) \partial_{z} \wedge \partial_{x}+\partial_{z}(g) \partial_{x} \wedge \partial_{y},
$$

i.e., we contract the differential 1-form $d g$ to $\partial_{x} \wedge \partial_{y} \wedge \partial_{z}$. Consider the toric surface $A_{n}$ given by $g(x, y, z)=x y-z^{n+1}$. We would like to express $\pi_{g}$ in the form (9). We see that it holds $\pi_{g}(x, y)=-(n+1) z^{n}, \pi_{g}(z, x)=x$ and $\pi_{g}(y, z)=y$. In this case $\Lambda$ is generated by $S_{1}:=(0,1), S_{2}:=(1,1)$ and $S_{3}:=(n+1, n)$, with the relation $S_{1}+S_{3}=(n+1) S_{2}$. We would like to find $p$ of the form (9), such that $p=\pi_{g}$. With a simple computation, we see that $p$ is of degree $-S_{2}$ :

$$
p\left(x^{\lambda_{1}}, x^{\lambda_{2}}\right)=f_{0}\left(\lambda_{1}, \lambda_{2}\right) x^{-S_{2}+\lambda_{1}+\lambda_{2}},
$$

where $f_{0}\left(S_{1}, S_{3}\right)=-(n+1)$. The function $f_{0}$ is with this completely determined by skew-symmetry and bi-additivity.

Let us now briefly recall the GIT quotient construction $\mathbb{A}^{n} / / G$ of an affine toric variety (see e.g. [11, Chapter 5]). Let $X$ be an affine toric variety without torus factors, i.e., given by the full-dimensional cone $\sigma=\left\langle a_{1}, \ldots, a_{N}\right\rangle \subset N_{\mathbb{R}}$. We have a short exact sequence

$$
0 \rightarrow M \stackrel{g}{\rightarrow} \mathbb{Z}^{\sigma(1)} \rightarrow \mathrm{Cl}(X) \rightarrow 0
$$


where $\mathrm{Cl}(X)$ is the class group of $X, \sigma(1)=N$ is the number of ray generators and $g$ is an injection map $g(R)=\left\langle R, a_{1}\right\rangle e_{1}+\cdots+\left\langle R, a_{N}\right\rangle e_{N}$, where $e_{j}, j=1, \ldots, N$ is the standard basis for $\mathbb{Z}^{N}$. We have $X=\mathbb{A}^{n} / / G$, where $G=\operatorname{Hom}_{\mathbb{Z}}\left(\mathrm{Cl}(X), k^{*}\right)$.

Remark 6. In the above GIT quotient construction we need the assumption that $k$ is algebraically closed. Moreover, the construction can be generalized to semiprojective toric varieties, if we take the GIT quotient of $\mathbb{A}^{n} \backslash Z$ for some exceptional set $Z$, which is $\emptyset$ in the case of affine toric varieties.

The map $g$ induce a semi-group isomorphism between $\Lambda \subset M$ and its image $\Lambda^{G}:=g(\Lambda)$. This map determines the isomorphism map of $k$-algebras

$$
G^{\prime}: k[\Lambda] \rightarrow k\left[x_{1}, \ldots, x_{N}\right]^{G},
$$

with $G^{\prime}\left(x^{R}\right)=x^{g(R)}:=x_{1}^{\left\langle R, a_{1}\right\rangle} \cdots x_{N}^{\left\langle R, a_{N}\right\rangle}$. Elements that lie in $\Lambda^{G}$ are $G$-invariant elements. Thus we have $X=\operatorname{Spec}\left(k\left[x_{1}, \ldots, x_{N}\right] / / G\right)=\operatorname{Spec}\left(k\left[x_{1}, \ldots, x_{N}\right]^{G}\right)$.

Proposition 5.6. For $\lambda, R \in M$ it holds that

$$
\lambda \in \cup_{j \in I} K_{a_{j}}^{R} \text { if and only if } g(\lambda) \in \cup_{j \in I} K_{e_{j}}^{g(R)} \text {, }
$$
$\mathbb{R}^{N}$.

where $I=\{1, \ldots, N\}$ and $K_{e_{j}}^{g(R)}$ are the convex sets (3) of the cone $\left\langle e_{1}, \ldots, e_{N}\right\rangle \subset$

Proof. By the definition of $g$ we know that $\left\langle g(\lambda), e_{j}\right\rangle=\left\langle\lambda, a_{j}\right\rangle$ and $\left\langle g(R), e_{j}\right\rangle=$ $\left\langle R, a_{j}\right\rangle$. For $g(\lambda) \in \cup_{j} K_{e_{j}}^{g(R)}$ there exists $j$ such that $\left\langle g(\lambda), e_{j}\right\rangle\left\langle\left\langle g(R), e_{j}\right\rangle\right.$ which means that there exists $j$ such that $\left\langle\lambda, a_{j}\right\rangle\left\langle\left\langle R, a_{j}\right\rangle\right.$, which is equivalent to $\lambda \in$ $\cup_{j} K_{a_{j}}^{R}$.

Let $A=k\left[\sigma^{\vee} \cap M\right]$ and $X=\operatorname{Spec}(A)$ be a toric variety without torus factors. Let $T_{k}=\operatorname{Spec}\left(k\left[\mathbb{Z}^{k}\right]\right)$ and $A_{k}=k\left[\Lambda \times \mathbb{Z}^{k}\right]\left(A_{0} \cong A\right)$. Every affine toric variety is of the form $X_{k}=\operatorname{Spec}\left(A_{k}\right)=X \times T_{k}$. Let $Y_{k}=\mathbb{A}^{N} \times T_{k}=\operatorname{Spec}\left(B_{k}\right)$, where $B_{k}=k\left[\mathbb{N}_{0}^{N} \times \mathbb{Z}^{k}\right]$ and $\mathbb{N}_{0}$ is the set of natural numbers with 0 . We define lattices $\widetilde{M}:=M \times \mathbb{Z}^{k}, \widetilde{N}:=N \times \mathbb{Z}^{k}$ and a map $g^{\prime}: \Lambda \times \mathbb{Z}^{k} \rightarrow \mathbb{N}_{0}^{N} \times \mathbb{Z}^{k}$ with

$$
g^{\prime}(\lambda, \mu)=(g(\lambda), \mu) .
$$

Definition 14. Let $(V,\{\cdot, \cdot\})$ be an affine Poisson variety and let $p: V \rightarrow W$ be a dominant map, where $W$ is an affine variety. If there exists a Poisson structure $\{\cdot, \cdot\}_{W}$ on $W$, such that for every $x \in V$,

$$
\{F, G\}_{W}(p(x))=\{\bar{F}, \bar{G}\}(x),
$$

for all $F, G \in \mathcal{O}(W)$ and for all extensions $\bar{F}, \bar{G}$ of $F \circ p$ and $G \circ p$, we call $\{\cdot, \cdot\}_{W}$ a reduced Poisson structure.

Proposition 5.7. Every Poisson structure $p$ on $X_{k}$ can be seen as a reduced Poisson structure $P$ on $Y_{k}$.

Proof. From Proposition 5.5 we know that every Poisson structure on $X_{k}$ is of the form

$$
p\left(x^{\lambda_{1}}, x^{\lambda_{2}}\right)=\sum_{i=0}^{d} f_{i}\left(\lambda_{1}, \lambda_{2}\right) x^{R_{i}+\lambda_{1}+\lambda_{2}},
$$

where $f_{i} \in \bar{C}_{(2)}^{2}\left(\Lambda \times \mathbb{Z}^{k},\left(\Lambda \times \mathbb{Z}^{k}\right) \backslash\left(-R_{i}+\left(\Lambda \times \mathbb{Z}^{k}\right)\right) ; k\right), R_{i} \in \widetilde{M}$. 
Now we construct a Poisson structure $P$ on a smooth affine variety $Y_{k}$ :

$$
P\left(x^{\lambda}, x^{\mu}\right)=\sum_{i=0}^{d} F_{i}(\lambda, \mu) x^{g^{\prime}\left(R_{i}\right)+\lambda+\mu},
$$

where $F_{i}$ has the property that $F_{i}\left(g^{\prime}\left(\lambda_{1}\right), g^{\prime}\left(\lambda_{2}\right)\right)=f_{i}\left(\lambda_{1}, \lambda_{2}\right)$, for each $i$.

STEP 1: Functions $F_{i}$ with the above property exist for each $i$ :

We choose $k+n$ linearly independent vectors $s_{1}, \ldots, s_{k+n} \in \Lambda \times \mathbb{Z}^{k}$ such that $s_{1}, \ldots, s_{k} \in 0 \times \mathbb{Z}^{k}$ and $s_{k+1}, \ldots, s_{k+n} \in \Lambda \times 0$. Note also that $f_{i}$ are completely determined by the values $f_{i}\left(s_{j}, s_{l}\right)$, for $1 \leq j<l \leq k+n$ by Remark 2 . Since $g^{\prime}$ is injective we can choose $F_{i} \in \bar{C}_{(2)}^{2}\left(\mathbb{N}_{0}^{N} \times \mathbb{Z}^{k} ; k\right)$, such that $F_{i}\left(g^{\prime}\left(s_{j}\right), g^{\prime}\left(s_{l}\right)\right)=$ $f_{i}\left(s_{j}, s_{l}\right)$, for $1 \leq j<l \leq k+n$.

Let $t_{1}, \ldots, t_{N-n} \in \mathbb{N}_{0}^{N}$ be chosen such that $s_{k+1}, \ldots, s_{k+n}, t_{1}, \ldots, t_{N-n}$ determine $\mathbb{R}$-basis of $\mathbb{R}^{N}$. We choose $F_{i}$ such that $F_{i}\left(t_{j}, t_{l}\right)=0$ for $1 \leq j, l \leq N-n$ and $F_{i}\left(s_{j}, t_{l}\right)=0$ for $j=1, \ldots, k+n$ and $l=1, \ldots, N-n$ (this will be important to prove the Jacobi identity for $P$ in Step 3). We easily see that it holds $F_{i}\left(g^{\prime}\left(\lambda_{1}\right), g^{\prime}\left(\lambda_{2}\right)\right)=$ $f_{i}\left(\lambda_{1}, \lambda_{2}\right)$.

STEP 2: $P$ is well defined:

That $P\left(x^{\lambda_{1}}, x^{\lambda_{2}}\right)$ is well defined it must for each $i$ hold that $F_{i}(\lambda, \mu)=0$ for $g^{\prime}(R)+\lambda+\mu \nsupseteq 0$. We need to check that this agrees with the property $F_{i}\left(g^{\prime}\left(\lambda_{1}\right), g^{\prime}\left(\lambda_{2}\right)\right)=f_{i}\left(\lambda_{1}, \lambda_{2}\right)$ : without loss of generality $\lambda_{1}, \lambda_{2} \in \Lambda \times 0$. We have $F_{i}\left(g\left(\lambda_{1}\right), g\left(\lambda_{2}\right)\right)=0$ for $g(R)+g\left(\lambda_{1}\right)+g\left(\lambda_{2}\right) \nsupseteq 0$ or equivalently for $g\left(\lambda_{1}+\right.$ $\left.\lambda_{2}\right) \in \mathbb{N}_{0}^{N} \backslash \mathbb{N}_{0}^{N}(-g(R))=\cup_{j \in I} K_{e_{j}}^{-g(R)}$, where $I=\{1, \ldots, N\}$. By Proposition 5.6 this is equivalent to $\lambda_{1}+\lambda_{2} \in \cup_{j \in I} K_{a_{j}}^{-R}$ and we indeed have $f_{i}\left(\lambda_{1}, \lambda_{2}\right)=0$ for $R+\lambda_{1}+\lambda_{2} \nsupseteq 0$.

STEP 3: $P$ satisfies the Jacobi identity:

We have $e_{3}(3)([p, p])\left(x^{\lambda_{1}}, x^{\lambda_{2}}, x^{\lambda_{3}}\right)=0$, since $p$ is a Poisson structure. Using Lemma 5.4 and the equalities $F_{i}\left(g^{\prime}\left(\lambda_{1}\right), g^{\prime}\left(\lambda_{2}\right)\right)=f_{i}\left(\lambda_{1}, \lambda_{2}\right)$ from Step 1, we see that $e_{3}(3)([P, P])\left(x^{g^{\prime}\left(\lambda_{1}\right)}, x^{g^{\prime}\left(\lambda_{2}\right)}, x^{g^{\prime}\left(\lambda_{3}\right)}\right)=0$. Since $e_{3}(3)[P, P] \in H_{(3)}^{3}\left(Y_{k}\right)$ we can use Proposition 4.7 and thus from the construction of $F_{i}$ in Step $1\left(F_{i}\left(t_{j}, t_{l}\right)=0\right.$ and $\left.F_{i}\left(s_{j}, t_{l}\right)=0\right)$ we immediately see that $e_{3}(3)[P, P]=0$. Thus the Jacobi identity is satisfied.

Let $\mathfrak{g}$ denote the differential graded Lie algebra $\left(\hbar C \cdot\left(A_{k}\right)[1]\right)[[\hbar]]$ and let $\mathfrak{h}$ denote the differential graded Lie algebra $\left(\hbar C \cdot\left(B_{k}\right)[1]\right)[[\hbar]]$.

Proposition 5.8. Let $\gamma\left(x^{\lambda_{1}}, x^{\lambda_{2}}\right):=\sum_{m \geq 1} \hbar^{m} \gamma_{m}\left(x^{\lambda_{1}}, x^{\lambda_{2}}\right) \in \mathfrak{h}^{1}$ be an MC element of a dgla $\mathfrak{h}$, where $\gamma_{1}$ is a Poisson structure on $Y_{k}$ of index $\left(g^{\prime}\left(R_{0}\right), \ldots, g^{\prime}\left(R_{d}\right)\right)$. Then $\gamma$ induces an $M C$ element $\widetilde{\gamma}\left(x^{\lambda_{1}}, x^{\lambda_{2}}\right):=\sum_{m \geq 1} \hbar^{m} \widetilde{\gamma}_{m}\left(x^{\lambda_{1}}, x^{\lambda_{2}}\right) \in \mathfrak{g}^{1}$ of the dgla $\mathfrak{g}$, where $\widetilde{\gamma}_{1}$ is a reduced Poisson structure on $\bar{X}_{k}$ of index $\left(R_{0}, \ldots, R_{d}\right)$.

Proof. We prove it just for $d=0$ and $k=0$ (i.e. for $\gamma_{1}$ of degree $R_{0}$ on a toric variety $X=X_{0}$ without torus factors). The rest follows easily, just the notation is more tedious.

We know that $\gamma_{m}\left(x^{\lambda_{1}}, x^{\lambda_{2}}\right)=\gamma_{0 m}\left(\lambda_{1}, \lambda_{2}\right) x^{m g(R)+\lambda_{1}+\lambda_{2}}$, where

$$
\gamma_{0 m} \in C^{2}\left(\mathbb{N}_{0}^{N}, \mathbb{N}_{0}^{N} \backslash \mathbb{N}_{0}^{N}(-m g(R)) ; k\right) .
$$

We define $\widetilde{\gamma}_{0 m}(\lambda, \mu):=\gamma_{0 m}(g(\lambda), g(\mu))$ and $\widetilde{\gamma}:=\sum_{m \geq 1} \hbar^{m} \widetilde{\gamma}_{m}\left(x^{\lambda}, x^{\mu}\right)$, where $\widetilde{\gamma}_{m}\left(x^{\lambda}, x^{\mu}\right)=\widetilde{\gamma}_{0 m}(\lambda, \mu) x^{m R+\lambda+\mu}$. 
First we need to check that $\widetilde{\gamma}\left(x^{\lambda}, x^{\mu}\right)=\sum_{m>1} \hbar^{m} \widetilde{\gamma}_{m}\left(x^{\lambda}, x^{\mu}\right)$ is well defined, i.e., if $m R+\lambda+\mu \nsupseteq 0$, then $\gamma_{0 m}(g(\lambda), g(\mu))=0$. This can be done as in Step 2 of Proposition 5.7 .

Looking only at $G$-invariant elements (i.e. $\lambda=g\left(\lambda^{\prime}\right)$ and $\mu=g\left(\mu^{\prime}\right)$ for some $\left.\lambda^{\prime}, \mu^{\prime} \in \Lambda\right)$ in the MC equation for $\gamma$ and using Lemma 5.4, we see that the MC equation also holds for $\widetilde{\gamma}$.

Theorem 5.9. Every Poisson structure $p$ on an affine toric variety can be quantized.

Proof. As above let $X_{k}$ denote an arbitrary affine toric variety. By Proposition 5.5 , $p$ is of the form $p\left(x^{\lambda_{1}}, x^{\lambda_{2}}\right)=\sum_{i=0}^{d} f_{i}\left(\lambda_{1}, \lambda_{2}\right) x^{R_{i}+\lambda_{1}+\lambda_{2}}$ for some $R_{i} \in \Lambda \times \mathbb{Z}^{k}$. By the construction in the proof of Proposition 5.7 this Poisson structure can be seen as a reduced Poisson structure of $P$ on $Y_{k}$ :

$$
P\left(x^{\lambda}, x^{\mu}\right)=\sum_{i=0}^{d} F_{i}(\lambda, \mu) x^{g^{\prime}\left(R_{i}\right)+\lambda+\mu},
$$

where the functions $F_{i}$ have the property that $F_{i}\left(g^{\prime}\left(\lambda_{1}\right), g^{\prime}\left(\lambda_{2}\right)\right)=f_{i}\left(\lambda_{1}, \lambda_{2}\right)$. Since $P$ is a Poisson structure on a smooth affine variety $Y_{k}$, we know by Corollary 5.3 that $P$ can be quantized. In other words there exists a one parameter deformation and by Lemma 5.1 we know that this correspond to an MC element $\gamma\left(x^{\lambda_{1}}, x^{\lambda_{2}}\right):=\sum_{m \geq 1} \hbar^{m} \gamma_{m}\left(x^{\lambda_{1}}, x^{\lambda_{2}}\right) \in \mathfrak{h}^{1}$, where $\gamma_{1}$ is of index $\left(g^{\prime}\left(R_{0}\right), \ldots, g^{\prime}\left(R_{d}\right)\right)$. By Proposition 5.8 we know that this give us an MC element

$$
\widetilde{\gamma}\left(x^{\lambda_{1}}, x^{\lambda_{2}}\right):=\sum_{m \geq 1} \hbar^{m} \widetilde{\gamma}_{m}\left(x^{\lambda_{1}}, x^{\lambda_{2}}\right) \in \mathfrak{g}^{1},
$$

where $\widetilde{\gamma}_{1}$ is a reduced Poisson structure on $X_{k}$ of index $\left(R_{0}, \ldots, R_{d}\right)$. By the construction we have $\widetilde{\gamma}_{1}=p$. Using again Lemma 5.1 we see that $p$ can be quantized.

\section{ACKNOWLEDGEMENTS}

This paper is part of my PhD thesis. I would like to thank to my advisor Klaus Altmann, for his constant support and for providing clear answers to my many questions. I am also grateful to Victor P. Palamodov, Giangiacomo Sanna and Arne B. Sletsjøe for useful discussions.

\section{REFERENCES}

[1] K. Altmann: Minkowski sums and homogenous deformation of toric varieties, Tohoku Math. J. 47 (1995), 151-184.

[2] K. Altmann: The versal deformation of an isolated, toric Gorenstein singularity, Invent. Math. 128 (1997), 443-479.

[3] K. Altmann: One parameter families containing three-dimensional toric Gorenstein singularities, Explicit birational geometry of 3-folds, London Math. Soc. Lecture Note Ser., vol. 281, Cambridge Univ. Press, Cambridge (2000), 21-50.

[4] K. Altmann, A. B. Sletsjøe: André-Quillen cohomology of monoid algebras, J. Alg. 210 (1998), 1899-1911.

[5] F. Bayen, M. Flato, C. Frønsdal, A. Lichnerowicz, D. Sternheimer: Deformation theory and quantization, Ann. of Phys. 111 (1978), 61-110 and 111-151.

[6] D. Barnette: A proof of the lower bound conjecture for convex polytopes Pac. J. of Math. 46, vol. 2 (1973), 349-354.

[7] M. Brion: The structure of the polytope algebra, Tohoku Math. J. 49 (1997), 1-32.

[8] D. Calaque, T. Pantev, B. Toën, M. Vaquié, G. Vezzosi: Shifted Poisson Structures and deformation quantization, arXiv:1506.03699 
[9] L. Cirio, G. Landi, R. J. Szabo: Algebraic deformations of toric varieties I: general constuctions, Adv. Math. 246 (2013), 33-88.

[10] L. Cirio, G. Landi, R. J. Szabo: Algebraic deformations of toric varieties II: noncommutative instantons, Adv. Theor. Math. Phys. 15 (2011), 1817-1907.

[11] D. A. Cox, J. B. Little, H.K. Schenk: Toric varieties, Graduate Studies in Mathematics 124, AMS (2011).

[12] C. Di Natale, E. Fatighenti, D. Fiorenza: Hodge theory and deformations of affine cones of subcanonical projective varieties, to appear in J. of London Math. Soc.

[13] M. Cirafici, A. Sikovics, R.J. Szabo: Cohomological gauge theory, quiver matrix models and Donaldson-Thomas theory, Nucl. Phys. B 809 (2009), 452-518.

[14] V. Dolgushev, D. Tamarkin, B. Tsygan: The homotopy Gerstenhaber algebra of Hochschild cochains of a regular algebra is formal, J. Noncommut. Geom. 1 (2007), iss. 1, 1-25.

[15] C. Frønsdal, M. Kontsevich: Quantization on Curves, Lett. Math. Phys. 79 (2007), 109-129.

[16] M. Gerstenhaber, S.D. Schack: A Hodge-type decomposition for commutative algebras, J. Pure Appl. Alg. 48 (1987), 229-247.

[17] L. Illusie: Complexe cotangent et déformations I,II, Lecture notes in Math, 239, 283 Springer (1971-1972).

[18] A. Iqbal, N.A. Nekrasov, A. Okounkov, C. Vafa: Quantum foam and topological strings, J. High Energy Phys. 0804 (2008), 011.

[19] M. Kontsevich: Deformation quantization of algebraic varieties, Lett. Math. Phys. 56 (2001), iss. $3,271-294$.

[20] M. Kontsevich: Deformation quantization of Poisson manifolds, Lett. Math. Phys. 66 (2003), iss. $3,157-216$.

[21] J.-L. Loday: Cyclic homology, Grundlehren der mathematischen Wissenschaften 301, Springer-Verlag, (1992).

[22] M. Manetti: The cotangent complex in characteristic 0 , available at: http://www1.mat.uniroma1.it/people/manetti/DT2011/marco2.pdf

[23] O. Mathieu, Homologies associated with Poisson structures, Deformation theory and symplectic geometry (Ascona 1996), Math. Whys. Stud., vol. 20, Kulwer Acad. Publ., Dordrecht (1997), 177-199.

[24] R.I. Michler: Hodge components of cyclic homology for affine quasi-homogenous hypersurfaces, Astérisque 226 (1994), 321-333.

[25] V.P. Palamodov: Infinitesimal deformation quantization of complex analytic spaces, Lett. Math. Phys. 79 (2007), iss. 2, 131-142.

[26] V.P. Palamodov: Associative deformations of complex analytic spaces, Lett. Math. Phys. 82 (2007), iss. 2, 191-217.

[27] H.C. Pinkham: Deformations of quotient surface singularities, Proc. Symp. Pure Math. 30, Part 1 (1977), 65-67.

[28] T. Saito: Parity in Bloch's conductor formula in even dimension, J. Théor. Nombres Bordeaux 16 (2004), 403-421.

[29] T. Schedler: Deformations of algebras in noncommutative geometry, Noncommutative Algebraic Geometry, Cambridge University Press (2016), Series: Math. Sc. Research Institute pub. 64.

[30] B. Totaro: Jumping of the nef cone for Fano varieties, J. Algebraic Geom. 21 vol. 2 (2012), 375-396.

[31] M. Van den Bergh: On global deformation quantization in the algebraic case, J. Alg. 315 (2007), 326-395.

[32] A. Yekutieli: Deformation quantization in algebraic geometry, Adv. Math. 198, (2005), 383432.

[33] A. Yekutieli: Twisted deformation quantization of algebraic varieties. Adv. Math. 268 (2015), 241-305.

Institut für Mathematik, Freie Universität Berlin, Berlin, Germany

E-mail address: filip@math.fu-berlin.de 